\title{
Searching for links between magnetic fields and stellar evolution
}

\section{A survey of magnetic fields in open cluster A- and B-type stars with FORS1 $1^{\star} \star \star \star$}

\author{
S. Bagnulo ${ }^{1}$, J. D. Landstreet ${ }^{2}$, E. Mason ${ }^{1}$, V. Andretta ${ }^{3}$, J. Silaj ${ }^{2}$, and G. A. Wade ${ }^{4}$ \\ 1 European Southern Observatory, Casilla 19001, Santiago 19, Chile \\ e-mail: sbagnulo@eso.org; emason@eso.org \\ 2 Physics \& Astronomy Department, The University of Western Ontario, London, Ontario, N6A 3K7, Canada \\ e-mail: jlandstr@astro.uwo.ca;jsilaj@uwo.ca \\ 3 INAF - Osservatorio Astronomico di Capodimonte, salita Moiariello 16, 80131 Napoli, Italy \\ e-mail: andretta@na.astro.it \\ 4 Department of Physics, Royal Military College of Canada, PO Box 17000, Station "Forces" Kingston, Ontario, K7K 7B4, Canada \\ e-mail: Gregg.Wade@rmc.ca
}

Received 19 September 2005 / Accepted 12 January 2006

ABSTRACT

Context. About $5 \%$ of upper main sequence stars are permeated by a strong magnetic field, the origin of which is still matter of debate.

Aims. With this work we provide observational material to study how magnetic fields change with the evolution of stars on the main sequence, and to constrain theory explaining the presence of magnetic fields in A and B-type stars.

Methods. Using FORS1 in spectropolarimetric mode at the ESO VLT, we have carried out a survey of magnetic fields in early-type stars belonging to open clusters and associations of various ages.

Results. We have measured the magnetic field of 235 early-type stars with a typical uncertainty of $\sim 100 \mathrm{G}$. In our sample, 97 stars are Ap or Bp stars. For these targets, the median error bar of our field measurements was $\sim 80 \mathrm{G}$. A field has been detected in about 41 of these stars, 37 of which were not previously known as magnetic stars. For the 138 normal A and B-type stars, the median error bar was $136 \mathrm{G}$, and no field was detected in any of them.

Key words. stars: magnetic fields - stars: chemically peculiar - stars: evolution - polarization - techniques: polarimetric

\section{Introduction}

The presence of strong $(\sim 1 \mathrm{kG})$ magnetic fields in some of the A- and B-type stars of the upper main sequence has been known for more than 50 years (Babcock 1947). These fields are almost invariably associated with a suite of other unusual characteristics, which include (a) specific angular momentum of the order of $10 \%$ or less of the typical value for normal main sequence stars of similar mass; (b) quite anomalous atmospheric chemical composition, which is to first approximation a function of effective temperature (and thus is apparently an atmospheric rather than a global feature); and (c) variation of the spectrum, light and magnetic field with the star's rotational period, that clearly indicate the presence of quite significant

* Based on observations made with ESO Telescopes at the Paranal Observatory under programme ID 068.D-0403, 070.D-0352, 272.D-5026, 073.D-0498, and 074.D-0488.

$\star \star$ Appendix is only available in electronic form at http://www. edpsciences.org abundance inhomogeneities and of a magnetic field not symmetric about the rotation axis.

Although considerable progress has been made in understanding the physical processes at work in these stars, many important problems remain unsolved. Among these are two major questions. First, although there is strong evidence (e.g., the stability of the observed fields, the lack of symptoms of Sunlike activity, and the lack of any important correlation of observed field strength with rotational angular velocity) that the observed fields are fossil fields, it is not yet clear how these fields evolve during the main sequence phase. Secondly, although it is believed that the basic mechanism leading to both chemical anomalies and to atmospheric inhomogeneities is the competition between gravitational settling, radiative levitation, and various hydrodynamic processes, the interplay of these processes is still very poorly understood.

In this situation, it is helpful to look to observations to guide physical theory. One kind of information about the magnetic Ap and Bp stars (hereafter referred to as magnetic 
Ap stars) that has been almost entirely lacking is the age of observed stars. Good age information would be very useful for discerning systematic evolutionary changes in field strength, chemical composition, rotation rates, etc. The general lack of useful age information about magnetic Ap stars occurs because almost all of the bright magnetic Ap stars are field stars. Even with the accurate parallaxes now available from the Hipparcos mission, the uncertainties in luminosity and effective temperature of Ap stars are large enough that placing them in the HR diagram only suffices to determine very roughly their stage of evolution (see Sect. 2.1 below).

The obvious way to obtain improved ages for magnetic Ap stars is to observe such stars in open clusters. Until recently such a study has not been possible because cluster Ap stars are mostly fainter than $V \approx 6$ or 7 , beyond the limit of accurate magnetic field measurements with available instruments. This situation has changed due to the development of a new generation of highly efficient spectropolarimeters and observing strategies, and their availability on large telescopes. In particular, the FORS1 spectropolarimeter on one of the ESO $8 \mathrm{~m}$ VLT telescopes has been shown to be a powerful tool for measuring fields in very faint Ap stars. It has recently been used to detect a field in a star of $V=12.88$, the faintest magnetic Ap in which a field has ever been detected (Bagnulo et al. 2004).

Another very important development has been the substantial increase in the number of probable magnetic Aps identified in clusters, particularly by the systematic surveys of Maitzen and his collaborators. Furthermore, the availability of very accurate proper motions for a very large number of stars from the Hipparcos mission and the Tycho- 2 catalogue has greatly facilitated the correct separation of cluster members from foreground and background stars.

The time is now clearly ripe for studying magnetic Ap stars in clusters to obtain for the first time a reasonably large sample of magnetic Ap stars of known absolute and evolutionary ages. We have started to carry out such a survey, using the FORS1 spectropolarimeter. The first stage of this survey is reported in this paper. Section 2 discusses the rationale and scope of this survey, in particular what the advantages are of studying open cluster stars compared to studying field stars, why FORS1 at the VLT is an ideal instrument for this survey, and how individual targets have been selected. Sections 3-6 describe how the magnetic field can be determined from observations of polarized spectra in terms of basic physics, observing strategy, data reduction, and $\left\langle B_{z}\right\rangle$ determination. These sections contain, for other FORS1 users, a detailed discussion of the optimized techniques we have developed for field measurement, and may be skipped by readers interested mainly in the observational results. In Sects. 7 and 8 we present and discuss the observations obtained during this survey. Conclusions are presented in Sect. 9.

\section{The scope of this survey}

\subsection{Why open cluster stars?}

The final goal of this series of papers will be to obtain ages, masses, and magnetic field strengths for a substantial number of Ap stars, and study whether there are evolutionary changes in the magnetic field strength. The magnetic (and sometimes the abundance) properties of a large sample of Ap stars in the field around the Sun are already known (e.g., Mathys 2004; Cowley \& Bord 2004). In principle, our evolutionary study could be based on these data of field stars. To justify the need of the present survey of magnetic cluster stars we first consider the extent to which ages and masses may be derived for the stars in the field.

Temperatures for many of field stars may be estimated using available $U B V, u v b y$, or Geneva photometry, together with calibrations provided by Stępień \& Dominiczak (1989), Hauck \& North (1993), and Napiwotzki et al. (1993). Luminosities require distances and bolometric corrections. Distances to many nearby Ap stars have recently been accurately determined by the Hipparcos project (see Gomez et al. 1998); for the Ap stars nearer than about $100 \mathrm{pc}$, the relative distance errors are less than about $10 \%$. The bolometric corrections required have been discussed by Lanz (1984). Thus, it is now possible to place a large number of Ap stars on the HR diagram, and, using the evolution tracks for stars of various masses, to estimate both the mass and the age of individual stars. This exercise has been carried out by Gomez et al. (1998), by Pöhnl et al. (2005), and by Kochukhov \& Bagnulo (2006) for a very large sample of nearby Ap stars, and by Hubrig et al. (2000) for a special sample of particularly slowly rotating magnetic Ap stars. In fact, as discussed below, this method is limited by large uncertainties.

Effective temperatures of magnetic Ap stars are still somewhat uncertain, more so than for normal main sequence stars because of the peculiar energy distributions of Ap stars (see Stępień \& Dominiczak 1989). A recent tabulation by Sokolov (1998) of effective temperatures for nearly 70 Ap stars, with comparisons to earlier determinations, suggests that the uncertainty in $T_{\text {eff }}$ typically about $5 \%$, or \pm 0.02 dex.

For Hipparcos parallaxes, the distance uncertainty at $100 \mathrm{pc}$ is about $8 \%$ (or about $0.035 \mathrm{dex}$ ), and increases with the distance itself. There is also an uncertainty in the appropriate bolometric correction (BC) to apply. First of all, there is an uncertainty of $\sim 0.1 \mathrm{mag}$ in the estimate of the BC of a normal A-type star (due in part to the uncertainty in $T_{\text {eff }}$ ). Then, for Ap stars, one has to apply a correction to the normal BC. According to Lanz (1984) this correction is uncertain by $\sim 0.2 \mathrm{mag}$. Finally, there is the uncertainty as to whether one should apply the LutzKelker correction, about $0.1 \mathrm{mag}$ at $100 \mathrm{pc}$. Taking these effects together, for nearby field stars, the uncertainty in $M_{\mathrm{bol}}$ is $\sim 0.3 \mathrm{mag}$, i.e., $\sim 0.1 \mathrm{dex}$ in $\log \left(L / L_{\odot}\right)$. These uncertainties, once converted in the $\left(\log t, M / M_{\odot}\right)$ plane, correspond to age uncertainties that may be as large as half the main sequence life.

Four examples of this kind of inference are shown in the upper panel of Fig. 1, for stars of about $2.5 M_{\odot}$ and $4.0 M_{\odot}$, each considered for an age near the beginning of its main sequence lifetime and near the end. Comparison is made with the tracks of Schaller et al. (1992) for $Z=0.02$. The star positions in the HR diagram, with their uncertainties (shaded boxes), are shown in the upper left panel. The deduced positions in the age-mass diagram are shown in the upper right panel. It is clear that the error boxes for observational characteristics of Ap stars translate into ages which are sufficiently uncertain that one cannot 

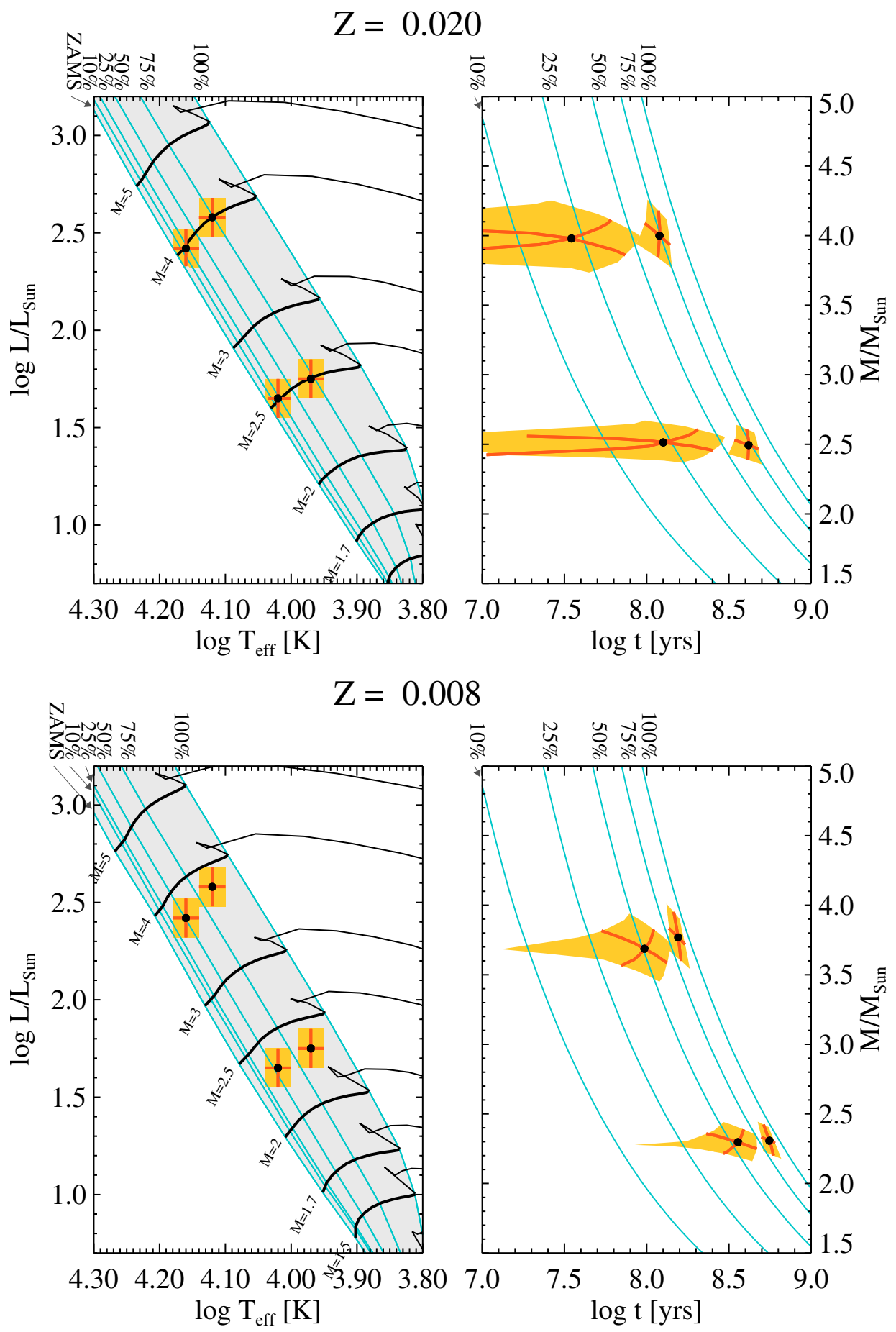

Fig. 1. Top panels: the position of a star in the HR diagram, and the star's position transformed into a diagram of age as a function of stellar mass, assuming that we know effective temperature $\pm 5 \%$ and luminosity \pm 0.1 dex. The transformation uses standard evolution tracks for $Z=0.02$ (Schaller et al. 1992); several fractional ages (fraction of main sequence life completed) are labelled. Bottom panels: the same transformation as in the top panels, but using tracks of metallicity $Z=0.008$ (Schaerer et al. 1993).

resolve at least the first half of the main sequence lifetime. The situation is somewhat better near the end of the main sequence life, where the isochrones are farther apart, but typically an age uncertainty of the order of $\pm 25 \%$ of the total main sequence lifetime is to be expected.

A further uncertainty in the transformation from the $\left(\log T_{\text {eff }}, \log \left(L / L_{\odot}\right)\right)$ to the $\left(\log t, M / M_{\odot}\right)$ plane comes from the fact that we do not know accurately the bulk chemical composition of any field Ap star, and thus we do not know what chemical composition should be assumed in the theoretical tracks used for the comparison. To estimate the size of this effect, we consider the range of abundances $([\mathrm{Fe} / \mathrm{H}])$ present in open clusters young enough to still have Ap stars (say $\log t<9$ ). Searching the WEBDA database (e.g. Mermilliod \& Paunzen 2003), we found that this range is of the order of $0.4 \mathrm{dex}$. This result is confirmed by examination of the younger 
clusters in the catalogue of Chen et al. (2003). This range of abundances may represent a reasonable estimate of the range of values of $[\mathrm{Fe} / \mathrm{H}]$ present in nearby field stars.

A change in $[\mathrm{Fe} / \mathrm{H}]$ of 0.4 dex corresponds to a change in metal abundance of about a factor of 2.5. This is just the difference in abundance between the evolution calculations with $Z=0.008$ and $Z=0.02$ provided by the Geneva group (Schaller et al. 1992; Schaerer et al. 1993). Repeating the transformation from the $\left(\log T_{\text {eff }}, \log \left(L / L_{\odot}\right)\right)$ to the $\left(\log t, M / M_{\odot}\right)$ plane for $Z=0.008$, we find the results shown in the lower panels of Fig. 1. (We have used the same values of $T_{\text {eff }}$ and $\log \left(L / L_{\odot}\right)$ as in the upper panels.) The effect of the uncertainty in bulk chemical composition is to add roughly another \pm 0.25 uncertainty to the deduced fraction of the main sequence life completed by a particular star. Overall, we see that knowledge of $T_{\text {eff }}$ and $\log \left(L / L_{\odot}\right)$ provides us with mass estimates accurate to about $\pm 10 \%$, but provides only poor age resolution, especially in the first half of the main sequence life (see also Fig. 4 of Kochukhov \& Bagnulo 2006).

The situation is substantially improved if the star is a member of a cluster or association. The absolute age of the star is then known with essentially the precision of the cluster age. The uncertainty of this number varies from cluster to cluster, mainly because of the difficulty of deciding exactly where to place the cluster turnoff. Typically the uncertainty in $\log t$ is of the order of \pm 0.2 dex, about $\pm 50 \%$ of the absolute age (see for example Castellani et al. 1992), although more accurate ages are sometimes reported (e.g. Carrier et al. 1999). This represents a very considerable improvement in absolute age, especially for a star in the early part of its main sequence life. If we know only position in the HR diagram, a star with an actual age of $10^{7} \mathrm{yr}$ and a main sequence lifetime of $10^{9} \mathrm{yr}$ would have an age uncertainty of the order of $3 \times 10^{8} \mathrm{yr}$, while the same star in a cluster would have an age uncertainty of roughly $3 \times 10^{6} \mathrm{yr}$. Only for a star in about the last third of its main sequence life is the age uncertainty not substantially improved by knowing that it is in a cluster. Another advantage of studying cluster stars is that one may determine the appropriate cluster bulk metallicity (and hence decide what evolution tracks to use for comparison) by studying the lower main sequence stars.

The masses of cluster stars can be determined with similar methods and similar (or better) accuracy than for local field stars. The values of $T_{\text {eff }}$ are known with about the same accuracy as for local field stars, and there are the same uncertainties for the bolometric correction. Hipparcos parallaxes are normally not available, but the value of luminosity is obtained from the observed $V$ magnitude together with the cluster's apparent distance modulus $\left(V-M_{V}\right)$. Recent determinations of distance modulus by main sequence fitting appear to achieve an accuracy of about \pm 0.2 mag (e.g., Robichon et al. 1999). This accuracy is generally obtained out to distances well beyond those few clusters for which accurate $( \pm 25 \%)$ parallaxes are available. Furthermore, if we know the cluster metallicity, we can decide which evolution tracks to use. The precision of the mass determination of cluster stars is about $\pm 10 \%$ if the bulk composition is not known, and somewhat better if it is.

Our conclusion is that an age derived using only the position in the HR diagram of an individual Ap star in the field is at present sufficiently uncertain to be of little value except for stars near the end of their main sequence lives (although important conclusions can still be drawn from statistical considerations: see Pöhnl et al. 2005 and Kochukhov \& Bagnulo 2006). In contrast, if the star is a member of a cluster of known age, it is possible to determine accurately both the mass and age (or fraction of main sequence life elapsed). Therefore, the study of Ap stars that are cluster members is of great value in understanding the temporal evolution of rotation, magnetic fields, and atmospheric chemistry in all magnetic Ap stars.

At present only a few cluster Ap star candidates are known to be magnetic. The total number of clusters for which magnetic observations had been published is eight, with a total of 13 stars surveyed. In addition, extensive surveys had been carried in for the Ori OB1 (Borra 1981) and Sco OB2 associations (Thompson et al. 1987). Accordingly, we have decided to carry out a survey of magnetic stars in open clusters to provide age information for a substantial sample of Ap stars.

\subsection{Why FORS1 at the VLT}

To decide on a suitable observing strategy, we must determine what field strength we wish to detect. Among Ap stars in the local field, it appears that the median root-mean-square observed line-of-sight field strength $\left\langle B_{z}\right\rangle$ is of the order of $300 \mathrm{G}$ (Bohlender \& Landstreet 1990). We assume that this will also be typical of cluster Ap stars. In order to detect such a small value, it is important for the survey to achieve measurement uncertainties of the order of $100 \mathrm{G}$ or less, as far as possible independent of the star's effective temperature and $v_{\mathrm{e}} \sin i$.

In principle, we may carry out a survey by searching either for visible Zeeman splitting of spectral lines in a simple intensity spectrum, or by searching for the circular polarization signature of a global field of simple structure. Although Mathys and collaborators (e.g., Mathys et al. 1997) have shown that Zeeman splitting may be detected in some tens of field stars, this requires quite special circumstances (i.e., $v_{\mathrm{e}} \sin i$ at most a few $\mathrm{km} \mathrm{s}^{-1}$, and field strength at least $2 \mathrm{kG}$ ) that are not met in most of the known magnetic Ap stars. Polarization measurement is generally a far more sensitive and broadly-applicable method of field detection than observation of Zeeman splitting.

Two main methods of measurement are currently in use for detecting the circular polarization produced by a non-zero value of $\left\langle B_{z}\right\rangle$. One method exploits the Zeeman polarization in metal lines (e.g., Babcock 1958; Preston \& Stępień 1968; Mathys \& Hubrig 1997; Wade et al. 2000; Elkin et al. 2003). Field measurement by this method usually relies on circular spectropolarimetry with a resolving power $R \geq 3 \times 10^{4}$. If the star is quite bright, with a $v_{\mathrm{e}} \sin i$ value smaller than, say, 15 or $20 \mathrm{~km} \mathrm{~s}^{-1}$ and a rich spectrum, field errors $\sigma_{B}$ as small as a few $\mathrm{G}$ can be achieved (Shorlin et al. 2002). On the other hand, the measurement uncertainty depends strongly on spectral type (which determines the number of usable lines and their intrinsic depths) and on $v_{\mathrm{e}} \sin i$. In observations collecting similar total numbers of photons, the standard error of field measurement can vary by a factor of order $10^{2}$. 
A second method employs the Zeeman polarization in the wings of the Balmer lines. These lines may be observed with quite low resolving power $\left(R \sim 10^{3}\right)$, using either interference filters (e.g., Borra \& Landstreet 1980) or a low-dispersion spectrograph (Bagnulo et al. 2002). Since the Balmer lines are intrinsically broad, the polarization signal may be a factor of 10 smaller than in the metal lines, and the best achievable standard errors are of order 30-50 G (Landstreet 1982). However, since the Balmer lines are always quite deep, and do not vary much in strength among $\mathrm{A}$ and $\mathrm{B}$ stars, and since the overall profile at $R \approx 10^{3}$ is hardly affected by rotation, this method can provide standard errors that are fairly uniform simply by surveying a sample of stars to a specified signal-to-noise ratio.

The (spectro-)polarimeters now in use make both kinds of field measurement. However, limitations imposed by low overall efficiency and (usually) modest telescope aperture have limited most of the $\left\langle B_{z}\right\rangle$ field measurements to stars not much fainter than $V=6$. This magnitude limit has effectively prevented any serious survey of magnetic fields in cluster Ap stars, as only a handful of clusters and associations have a significant number of Ap stars brighter than $V=6$. With a limiting magnetic around $V=6$, one is restricted to clusters and associations not much more than $100 \mathrm{pc}$ away, since the absolute magnitude of an Ap star is typically in the range of $M_{V} \sim-1$ to +1 . Only half a dozen clusters are this near.

The development of the FORS1 spectropolarimeter for the ESO Very Large Telescope (VLT) has changed the situation dramatically. FORS1 incorporates a multi-object low dispersion spectrograph with polarizing optics. In polarimetric mode, spectra with $R \sim 10^{3}$ may be obtained for up to nine objects simultaneously in a field $7^{\prime}$ square. Bagnulo et al. (2002) have shown that FORS1 can be used very effectively as a Balmer-line polarimeter for field measurement. Since this instrument is mounted on an $8 \mathrm{~m}$ telescope, and has a very high throughput, the limiting magnitude is much fainter than with earlier instruments. Bagnulo et al. (2004) have used FORS1 to detect a field in a star of $V \approx 13$ in $2.8 \mathrm{~h}$ of observation, and showed that it is possible to reach a precision of $\sigma_{B} \sim 10^{2} \mathrm{G}$ in about $1 \mathrm{~h}$ of integration at $V=10$. With a limiting magnitude of 10 or even fainter, we can survey clusters and associations out to several hundred pc, and the number of clusters that can be studied rises to roughly 100. In addition Bagnulo et al. (2002) have shown that the low resolution does not prevent the use of metal lines to measure the magnetic field in stars with rich metallic spectra. This fact may be used to increase the sensitivity of field measurements in some stars. A third advantage of FORS1 is that in clusters, it is often possible to observe several stars simultaneously. We have used this capability to observe multiple Ap candidate stars, but also non-Ap cluster members in the hopes of making serendipitous field detections.

This survey is biased toward stars with longitudinal field of absolute value larger than about 200-300 G, but is not biased with respect to $v \sin i$. Any bias with respect to spectral type is primarily a feature of the previous classification programmes that have identified candidate magnetic Ap stars in clusters, rather than a feature imposed by an instrumental field detection sensitivity that depends on the star's spectroscopic features.

\subsection{The target list}

Two problems arise in the selection of a list of suitable targets for a survey of magnetic fields in cluster stars: (a) identification of candidate Ap stars in the field of individual clusters (most of the known Ap stars are field stars) and (b) determination of cluster membership.

Numerous studies have identified probable Ap members of clusters and associations. Surveys for Ap/Bp cluster members based on low-dispersion spectroscopy were reported by Hartoog (1976, 1977), and by Abt (1979). Probable Ap stars have also been reported from a number of studies of individual clusters (e.g. Dachs \& Kabus 1989). Furthermore, the Michigan Spectral Survey (e.g. Houk \& Smith-Moore 1988) has provided uniform MK classifications for a large number of HD stars in the fields of clusters south of $\delta=+5^{\circ}$. This allowed us to select some Ap stars that are possible cluster members.

Another important source of identifications of cluster Ap stars has been the use of photometric indices that are sensitive to a broad depression near $5200 \AA$ in the energy distribution of most Ap stars (e.g., Kupka et al. 2003). In particular, Maitzen and collaborators (e.g., Maitzen 1993) have developed a narrow band photometric index $(\Delta a)$ which is reasonably sensitive to the spectral peculiarities of Ap stars with effective temperatures in the range $\sim 8000-14000 \mathrm{~K}$. Maitzen's group has systematically obtained $\Delta a$ photometry of many clusters to identify Ap stars. The $\Delta a$ system has also been used by Joncas \& Borra (1981) to search for Ap stars in the Orion OB1 association.

It is also known that the $Z$ index, which can be computed for the many cluster stars for which Geneva photometry is available, is a powerful discriminant of Ap stars in approximately the same temperature range as the $\Delta a$ index (e.g. Kramer \& Maeder 1980; Hauck \& North 1982).

We have made extensive use of the WEBDA cluster database (e.g. Mermilliod \& Paunzen 2003), Simbad, and a catalogue of candidate cluster Ap stars by Renson (1992).

Most of the surveys discussed above have made a serious effort to determine cluster memberships, mainly on the basis of spatial location and apparent magnitude. However, recent work based on and stimulated by the Hipparcos astrometric space mission has led to a major expansion in the available data on parallaxes and proper motions. Hipparcos parallaxes (e.g. Gomez et al. 1998) provide a valuable membership discriminant out to about $300 \mathrm{pc}$ for many cluster stars. Even more importantly, the Tycho (Høg et al. 1998) and Tycho-2 (Høg et al. 2000) proper motion catalogues now provide powerful tests of membership out to nearly one kpc, for a much larger number of stars, as demonstrated for example by Robichon et al. (1999), de Zeeuw et al. (1999), and Dias et al. (2001).

Using the resources discussed above, a database containing a variety of information on more than 200 suspected Ap cluster members in more than 70 clusters and associations was constructed and used for the selection of the targets. In general, we have given highest priority to stars which appear to be probable Ap stars, and probable cluster members, but we have also observed a number of stars for which at least one of these criteria was uncertain. Up to the present time, we have been able 
to observe (or occasionally find in the literature) magnetic observations of about $1 / 3$ of the stars in our database.

Cluster membership will be discussed in the second paper in this series (Landstreet et al., in preparation, hereafter referred to as Paper II), where we will analyze the astrophysical and evolutionary results of our survey in more detail. The present paper focuses on the magnetic observations themselves.

\section{The basic formulae}

The diagnostic tool for stellar magnetic fields used in this work has been proposed by Bagnulo et al. (2002). Here we review and elaborate this method.

The mean longitudinal magnetic field $\left\langle B_{z}\right\rangle$, i.e., the component of the magnetic field along the line of sight averaged over the visible stellar disk, can be measured through the analysis of the circular polarization of spectral lines. In the weak field regime (i.e., when the Zeeman splitting is small compared to the line intrinsic broadening) we have (e.g. Landstreet 1982)

$\frac{V}{I}=-g_{\mathrm{eff}} C_{z} \lambda^{2} \frac{1}{I} \frac{\mathrm{d} I}{\mathrm{~d} \lambda}\left\langle B_{z}\right\rangle$

where $g_{\text {eff }}$ is the effective Landé factor ( 1 for $\mathrm{H}$ Balmer lines, see Casini \& Landi Degl'Innocenti 1994), $V$ is the Stokes parameter which measures the circular polarization, $I$ is the total (unpolarized) intensity, $\lambda$ is the wavelength expressed in $\AA$, $\left\langle B_{z}\right\rangle$ is the longitudinal field expressed in Gauss, and

$C_{z}=\frac{e}{4 \pi m_{\mathrm{e}} c^{2}} \quad\left(\simeq 4.67 \times 10^{-13} \AA^{-1} \mathrm{G}^{-1}\right)$

where $e$ is the electron charge, $m_{\mathrm{e}}$ the electron mass, and $c$ the speed of light. For a typical A-star atmosphere, the weak-field approximation holds for field strength $\lesssim 1 \mathrm{kG}$ for metal lines, and up to $\sim 10 \mathrm{kG}$ for $\mathrm{H}$ lines.

A least-squares technique can be used to derive the longitudinal field via Eq. (1). We minimise the expression

$\chi^{2}=\sum_{i} \frac{\left(y_{i}-\left\langle B_{z}\right\rangle x_{i}-b\right)^{2}}{\sigma_{i}^{2}}$

where, for each spectral point $i, y_{i}=(V / I)_{i}, x_{i}=$ $-g_{\text {eff }} C_{z} \lambda_{i}^{2}(1 / I \times \mathrm{d} I / \mathrm{d} \lambda)_{i}$, and $b$ is a constant. For each spectral point $i$, the derivative of Stokes $I$ with respect to wavelength is evaluated as

$\left(\frac{\mathrm{d} I}{\mathrm{~d} \lambda}\right)_{\lambda=\lambda_{i}}=\frac{\mathcal{N}_{i+1}-\mathcal{N}_{i-1}}{\lambda_{i+1}-\lambda_{i-1}}$

where $\mathcal{N}_{i}$ is the photon count at wavelength $\lambda_{i}$. The estimates for the errors on the parameters $\left\langle B_{z}\right\rangle$ and $b$ are given by the diagonal elements of the inverse of the $\chi^{2}$ matrix (see, e.g., Bevington 1969).

The application of a least-squares technique is justified if

$\left\langle B_{z}\right\rangle \sigma_{x_{i}} \ll \sigma_{y_{i}}$

(see, e.g., Bevington 1969), where

$\sigma_{x_{i}}^{2}=\left(-g_{\mathrm{eff}} C_{z} \lambda_{i}^{2}\right)^{2}\left(\left(-\frac{1}{I^{2}}\right)^{2}\left(\frac{\mathrm{d} I}{\mathrm{~d} \lambda_{i}}\right)^{2} \sigma_{I}^{2}+\left(\frac{1}{I}\right)^{2} \sigma_{\mathrm{d} I / \mathrm{d} \lambda}^{2}\right)$ and

$\sigma_{y_{i}}^{2}=\sigma^{2}\left[(V / I)_{i}\right]$.

For the stars studied in this work, we have verified that, at all observed wavelengths, Eq. (5) is verified.

\section{Instrument and instrument setup}

The Focal Reducer/Low Dispersion Spectrograph FORS1 of the ESO VLT is a multi-mode instrument equipped with polarimetric optics. The instrument characteristics and performances are outlined by Seifert et al. (2000) and in the FORS1/2 User Manual (VLT-MAN-ESO-13100-1543). In this survey FORS1 has been used as a low resolution spectropolarimeter.

\subsection{Instrument mode}

Spectropolarimetry with FORS1 can be performed in two different observing modes: fast mode and fims mode. Fast mode is used for observations of individual objects that can be acquired "fast" with a simple centering on the slit. Fims mode permits one to place slitlets on up to nine targets within a $6.8^{\prime} \times 6.8^{\prime}$ field of view (multi-object spectropolarimetry). Whenever our main targets were in a field including other stars of similar magnitude, we observed in fims mode. In this way, in addition to polarized spectra of Ap stars, we obtained polarized spectra of numerous normal A and B-type cluster stars.

\subsection{Grism choice}

We have used the grisms $600 \mathrm{~B}$ and $600 \mathrm{R}$, which cover the spectral ranges $3450-5900 \AA$, and 5250-7450 $\mathrm{A}$, respectively. Grism $600 \mathrm{~B}$ covers all $\mathrm{H}$ Balmer lines from $\mathrm{H} \beta$ down to the Balmer jump; grism $600 \mathrm{R}$ covers $\mathrm{H} \alpha$. Both grisms have 600 grooves $\mathrm{mm}^{-1}$, and yield dispersions of $1.20 \AA$, and $1.08 \AA$ per pixel, respectively. With a 1 " slit width they provide spectral resolving powers of 780 and 1160, respectively.

For this study, grism $600 \mathrm{~B}$ seems a better choice than $600 \mathrm{R}$. Although $\mathrm{H} \alpha$ is more sensitive to the magnetic field than individual $\mathrm{H}$ Balmer lines at shorter wavelengths (the Zeeman effect depends quadratically upon wavelength), the combined analysis of several Balmer lines from $\mathrm{H} \beta$ down to the Balmer jump leads to a smaller error bar than the analysis of $\mathrm{H} \alpha$ only. Furthermore, the flux of an A-type stars is larger in the blue than in the red, therefore the same SNR can be reached with a shorter integration time using grism $600 \mathrm{~B}$ rather than grism $600 \mathrm{R}$. On the other hand, it should be noted that the useful field of view in fims mode depends on the spectral range that one wishes to cover: the larger the spectral range that one needs to observe, the smaller the spatial region where one can place slitlets. Hence, in fims mode, grism $600 \mathrm{R}$ offers more flexibility than grism $600 \mathrm{~B}$, as its useful wavelength coverage corresponds to $\mathrm{H} \alpha$ only, which does not put a strong constraint on the field of view. In (ESO period) P68 we used grism 600 R (in combination with the order separation filter GG 435). In P70, P72, P73, and P74, we used grism $600 \mathrm{~B}$ (with no order separation filter). 
For most of the observations, we have used a slit width of $0.5^{\prime \prime}$ or $0.8^{\prime \prime}$.

\section{3. $C C D$ readout}

In polarimetric mode, overheads represent a significant fraction of the total telescope time. Ultra-high SNR observations (needed to reach the wanted uncertainty of $\sim 100 \mathrm{G}$ ) require multiple exposures, hence, multiple CCD readouts. We minimized the CCD readout time as follows. In case of observations of individual stars (fast mode) we used a windowed read-out mode of $2048 \times 400$ pixels. In case of multi-object observations (fims mode) where the full size of the CCD was needed, we used a 4-port readout. The lowest available gain was selected, as to maximise the actual electron capacity, hence, the SNR that can be reached with each single exposure.

\section{Data reduction}

\subsection{Frame pre-processing, spectrum extraction and wavelength calibration}

The data have been reduced and optimally extracted using standard IRAF routines. All the science frames have been bias subtracted with the corresponding master bias obtained from a series of five frames taken the morning after the observations. No flat fielding procedure has been applied to our data. By performing reduction experiments with and without flat-fielding, we have verified that flat fielding does not influence significantly the final computation of the Stokes profiles. In fact, flat fields obtained with the grism $600 \mathrm{~B}$ are severely affected by internal reflections from the Longitudinal Atmospheric Dispersion Corrector (LADC). Frames obtained in fims mode were read out in four ports. The CCD is thus divided in four quadrants, each of them characterized by its own bias level and gain. In order to compensate for the different gains, we multiplied each science frames by the ratio of an imaging screen flat read out in one port and an imaging screen flat read out in four ports.

When extracting the spectra we found that the use of standard extraction apertures ( $\sim 10$ pixels width) introduced artefacts into the Stokes $V$ spectrum. This problem was solved using apertures as large as $\sim 4-5$ times the spatial FWHM of the spectrum, i.e., typically 50 pixels width. Apertures that are not symmetric about the flux peak have been used for stars close to the edge of the slit. This occurred occasionally for targets observed in fims mode. We used a high-order $(\sim 15)$ Legendre fitting function to trace the spectrum ${ }^{1}$. Lower order functions were used only in the case of low SNR secondary targets (typically in frames obtained in fims mode) and/or in the case of spectra the length of which occupied just a fraction of the CCD (again in fims frames, depending on the positioning of the slitlets).

Sky subtraction was performed differently for spectra obtained in fast and fims mode. In the first case the sky subtraction

\footnotetext{
1 The high order of the fitting function is justified by the high SNR that makes it possible to trace very accurately the spectrum across the CCD.
}

was performed selecting symmetric regions on the left and right side of each spectrum (typically between pixel 40 and 50 from the central peak), and fitting those with a Chebyshev polynomial. In the case of data obtained in fims mode, whenever the star was not positioned at the center of the slitlet, the sky was estimated on just one region at one side of the spectrum. In fact, we found that sky-subtraction is not critical in the sense that it does not significantly affect the final results. In some cases, we preferred not to perform sky-subtraction at all, because of the presence of LADC reflections close to the spectrum.

The FORS1 calibration plan includes wavelength calibrations frames obtained at all retarder waveplate positions used for the science. However, we found that the best and safest strategy is to use, for a complete set of science data, just a single wavelength calibration frame, and not match science and wavelength calibration frames according to their retarder waveplate angles. We found that this latter method occasionally introduces spurious polarization signals.

Wavelength calibration typically led to RMS scatter of $\sim 0.1$ pixels and maximum error of $\leq 20 \mathrm{~km} \mathrm{~s}^{-1}$. The fine tuning of wavelength calibration based on night sky lines could not be performed. Therefore the accuracy of the wavelength calibration is restricted by instrument flexures, which are expected to be less than 1 pixel up to a zenith distance of $60^{\circ}$ (see FORS1/2 User Manual). Numerical tests show that this is of negligible impact on the determination of the mean longitudinal magnetic field, using the method described by Bagnulo et al. (2002) and elaborated below.

\subsection{Obtaining stokes $V$}

Circular polarization measurements are performed by inserting the quarter-wave plate and the Wollaston prism into the optical path. A combination of exposures taken at different waveplate orientations allows one to minimise the contributions of spurious (instrumental) polarization. The FORS user manual explains that instrumental polarization cancels out to the first order if Stokes $V$ is obtained from

$\frac{V}{I}=\frac{1}{2}\left\{\left(\frac{f^{\mathrm{o}}-f^{\mathrm{e}}}{f^{\mathrm{o}}+f^{\mathrm{e}}}\right)_{\alpha=-45^{\circ}}-\left(\frac{f^{\mathrm{o}}-f^{\mathrm{e}}}{f^{\mathrm{o}}+f^{\mathrm{e}}}\right)_{\alpha=+45^{\circ}}\right\}$

where $f^{\mathrm{o}}$ and $f^{\mathrm{e}}$ are the ordinary and extraordinary beams, respectively, and $\alpha$ is the position angle of the retarder waveplate (in fact, note that the FORS1/2 manual gives the formula with the opposite sign). Another possibility is to obtain Stokes $V$ from

$\frac{V}{I}=\frac{r-1}{r+1}$

where

$r^{2}=\frac{\left(f^{\mathrm{o}} / f^{\mathrm{e}}\right)_{\alpha=-45^{\circ}}}{\left(f^{\mathrm{o}} / f^{\mathrm{e}}\right)_{\alpha=+45^{\circ}}}$

(e.g., Donati et al. 1997). We have verified that Eqs. (7) and (8) give consistent results both for Stokes $V$ and $\left\langle B_{z}\right\rangle$. 
The error bar associated with the Stokes $V / I$, computed via Eq. (7) is

$$
\begin{aligned}
\sigma^{2}[(V / I)]= & \left(\left(\frac{f^{\mathrm{e}}}{\left(f^{\mathrm{o}}+f^{\mathrm{e}}\right)^{2}}\right)^{2} \sigma_{f^{\mathrm{o}}}^{2}+\left(\frac{f^{\mathrm{o}}}{\left(f^{\mathrm{o}}+f^{\mathrm{e}}\right)^{2}}\right)^{2} \sigma_{f^{\mathrm{e}}}^{2}\right)_{\alpha=-45^{\circ}} \\
& +\left(\left(\frac{f^{\mathrm{e}}}{\left(f^{\mathrm{o}}+f^{\mathrm{e}}\right)^{2}}\right)^{2} \sigma_{f^{\mathrm{o}}}^{2}+\left(\frac{f^{\circ}}{\left(f^{\mathrm{o}}+f^{\mathrm{e}}\right)^{2}}\right)^{2} \sigma_{f^{\mathrm{e}}}^{2}\right)_{\alpha=+45^{\circ}} .
\end{aligned}
$$

Equation (9) can be simplified as follows. Let us assume

$\left(\sigma_{f^{\circ}}^{2}\right)_{\alpha= \pm 45^{\circ}}=\left(\sigma_{f^{\mathrm{e}}}^{2}\right)_{\alpha= \pm 45^{\circ}}=\left(f^{\mathrm{o}}\right)_{\alpha= \pm 45^{\circ}}=\left(f^{\mathrm{e}}\right)_{\alpha= \pm 45^{\circ}}=\mathcal{N}$

where $\mathcal{N}$ is an estimate of the photon count in a given wavelength range. For $m$ pairs of observations (i.e., one exposure at $\alpha=45^{\circ}$, and one exposure at $\alpha=-45^{\circ}$, repeated $m$ times), Eq. (9) becomes

$$
\sigma^{2}[(V / I)]=\frac{1}{4 m \mathcal{N}} .
$$

Equation (10) simply states that the error bar on $V / I$ decreases as $1 / \sqrt{\mathcal{N}}$. The polarization in a given wavelength range can be measured with an $0.1 \%$ uncertainty if at least $2.5 \times 10^{5}$ photons are accumulated both in the ordinary and in the extraordinary beam, and in each of the two exposures of a pair.

In order to detect weak magnetic fields $(\$ 300 \mathrm{G})$ with the technique used in this work, one has to obtain ultra high SNR $\left(\gtrsim 1500 \AA^{-1}\right)$ observations. Even with a $8 \mathrm{~m}$ telescope, this can be achieved only on relatively bright stars ( $V \lesssim 13$, if we limit the shutter time to $\$ 2 \mathrm{~h}$ ). Due to the limited CCD well capacity, multiple exposures have to be taken. From a practical point of view, one has to set the exposure time to a value that maximises the photon count without risk of CCD saturation (e.g., by adjusting the exposure time to get a peak count of 30000 ADU per pixel), and then take several pairs of exposures with the retarder waveplate at the $+45^{\circ}$ and $-45^{\circ}$ positions. Equation (10) can be explicitly expressed in terms of ADU in the following way. Let us define $M$ as the ADU per pixel and $g$ as the number of electrons per ADU, so that actual photon count $\mathcal{N}$ is given by $g M$. Let us also define $\mathcal{A}_{\mathrm{s}}$ as the ratio between the ADU integrated in a pixel column along the direction perpendicular to the dispersion, and the peak ADU in the central pixel. The error bar on the circular polarization measured in the wavelength interval $\Delta \lambda$ covered by 1 pixel is given by

$$
\sigma^{2}[(V / I)](\Delta \lambda)=\frac{1}{4 m g \mathcal{A}_{\mathrm{s}} M_{\max }}
$$

where $M_{\max }$ is the peak ADU.

Recalling the properties of a Gaussian, we can write $\mathcal{A}_{\mathrm{s}}=$ 1.065 FWHM. With a plate scale of $0.2^{\prime \prime}$ per pixel, as in the case of FORS 1 , and with $0.8^{\prime \prime}$ seeing, $\mathcal{A}_{\mathrm{s}} \simeq 4$. Assuming $g=$ 2.9 (a typical value for the FORS1 "low gain" readout mode), and setting as a peak value $M_{\max }=30000 \mathrm{ADU}$, we get

$\sigma[(V / I)](\Delta \lambda) \simeq 8 \times 10^{-4} \frac{1}{\sqrt{m}}$

Equation (11) allows one to calculate the number of pairs of exposures that are needed to measure $V / I$ with a given error bar. For instance, with a pair of exposures at half the CCD full well, one gets an error bar close to $0.1 \%$. To go below the threshold of an $0.01 \%$ error bar (if possible at all) one should obtain 64 pairs of exposures.

We found that measuring $V / I$ with an accuracy of a few units in $10^{-4}$ per $\AA$ in the continuum near to $\mathrm{H} \beta$ allowed us to measure magnetic fields with an error bar between 50 and $100 \mathrm{G}$. Therefore, we decided that our observing strategy would be based on a series of four pairs of exposures (following the sequence $\alpha=+45^{\circ},-45^{\circ},-45^{\circ},+45^{\circ}$, etc.). However, we could limit the number of pairs of exposure to four only when we obtained telescope time in visitor mode, which allowed us to optimize the exposure time based on the weather conditions. During P72 and P73 we were allocated telescope time in service mode, and were forced to set the exposure time to conservatively low values to be sure to avoid CCD saturation. In these cases the number of pairs of exposures was increased from four to six or eight to guarantee a sufficient SNR.

Thought must be given to the ratio between shutter time and overhead time, as the latter ( $20 \mathrm{~min}$ for a series of 4 pairs of exposures) may represent a substantial fraction of the total time for a single pointing. Using grism $600 \mathrm{~B}$ with a $0.5^{\prime \prime}$ slit width, we can obtain a peak ADU count of 20000 in about 10 min shutter time for a $V=11-12$ A-type star (depending on the weather conditions), and in just $1 \mathrm{~min}$ for $V=8.5-9.5$.

\subsection{The use of a $\sigma$-clipping algorithm}

The simplest method to obtain $V / I$ is to add up all spectra obtained in the same beam and with same retarder waveplate position, and then use Eq. (7). However, reduction products can be improved by using a $\sigma$-clipping algorithm as follows. For each wavelength step one calculates

$$
\begin{gathered}
\left(\frac{V}{I}\right)_{i j}=\frac{1}{2}\left\{\left(\frac{f_{i}^{\mathrm{o}}-f_{i}^{\mathrm{e}}}{f_{i}^{\mathrm{o}}+f_{i}^{\mathrm{e}}}\right)_{\alpha=-45^{\circ}}-\left(\frac{f_{j}^{\mathrm{o}}-f_{j}^{\mathrm{e}}}{f_{j}^{\mathrm{o}}+f_{j}^{\mathrm{e}}}\right)_{\alpha=+45^{\circ}}\right\} \\
i, j=1,2, \ldots, m
\end{gathered}
$$

where $m$ is the total number of pairs of observations. Then, one calculates the median $(\widehat{V / I})$ from the $m^{2}(V / I)_{i j}$ values, and the median absolute deviation (MAD), i.e., the median of the distribution

$\left|\left((V / I)_{i j}-(\widehat{V / I})\right)\right|$

Setting $\sigma=1.48$ MAD (e.g., Huber 1981, pp. 107-108), those $(V / I)_{i j}$ values for which

$$
\left|(V / I)_{i j}-(\widehat{V / I})\right|>\mathcal{K} \sigma
$$

are rejected (we typically adopted $\mathcal{K}=3$ ). The procedure is iterated until no points are rejected, but from the second iteration on, the median $(\widehat{V / I})$ is replaced by the weighted average

$$
\overline{(V / I)}=\sum_{i j}(V / I)_{i j} \sigma^{-2}\left[(V / I)_{i j}\right] / \sum_{i j} \sigma^{-2}\left[(V / I)_{i j}\right],
$$

where the sum is obviously extended over the $K(V / I)_{i j}$ values that have not been rejected by the $\sigma$-clipping algorithm $(K \leq$ $\left.m^{2}\right)$, and $\sigma^{2}\left[(V / I)_{i j}\right]$ is given by Eq. (9). 
Table 1. $\left\langle B_{z}\right\rangle$ measurements of the $V=6.1$ Ap star HD $94660=$ KQ Vel. Date, UT, and signal to noise ratio (SNR) are calculated as explained in the caption of Table A.3. Observations obtained in P66 had been already published by Bagnulo et al. (2002).

\begin{tabular}{lcclllll}
\hline \hline & & & \multicolumn{1}{c}{$\left\langle B_{z}\right\rangle(\mathrm{G})$} & \multicolumn{2}{c}{$\left\langle B_{z}\right\rangle(\mathrm{G})$} & \multicolumn{2}{c}{$\left\langle B_{z}\right\rangle(\mathrm{G})$} \\
Period & DATE & UT & Balmer lines & metal lines & full spectrum & SNR & Grism \\
\hline P66 & 2001-03-22 & $23: 54: 00$ & $-2085 \pm 85$ & $-2100 \pm 100$ & $-2260 \pm 65$ & 1550 & $600 \mathrm{~B}$ \\
P68 & 2002-02-04 & $08: 43: 13$ & $-2335 \pm 57$ & $-2141 \pm 52$ & $-2226 \pm 37$ & 2100 & $600 \mathrm{~B}$ \\
P68 & 2002-02-04 & $08: 56: 09$ & $-2083 \pm 75$ & $-2516 \pm 34$ & $-2439 \pm 31$ & 2150 & $600 \mathrm{R}$ \\
P70 & 2003-02-08 & $09: 41: 49$ & $-2574 \pm 57$ & $-2051 \pm 48$ & $-2260 \pm 38$ & 2150 & $600 \mathrm{~B}$ \\
P74 & 2005-01-30 & $09: 33: 45$ & $-2432 \pm 50$ & $-2002 \pm 43$ & $-2190 \pm 32$ & 2300 & $600 \mathrm{~B}$ \\
\hline
\end{tabular}

If no value in the $m^{2}$ set of $(V / I)_{i j}$ pairs has been rejected, and if we assume that the errors given by Eq. (9) are approximately equal for all spectra, we can estimate the error bar of $\overline{(V / I)}$ as:

$\left\{\sigma^{2}[\overline{(V / I)}]\right\}^{\prime} \simeq \frac{1}{m} \sigma^{2}[(V / I)]$.

An alternative estimate of the same error bar can be obtained as follows:

$\left\{\sigma^{2}[\overline{(V / I)}]\right\}^{\prime \prime} \simeq \frac{1}{m^{2}(m-1)} \sum_{i j}\left[(V / I)_{i j}-\overline{(V / I)}\right]^{2}$.

In practice, at each wavelength step, we adopt the error bar given by the maximum among the values obtained from Eqs. (14) and (15). The above two expressions will be derived in detail in Appendix A, together with more general expressions valid for the case of one or more exposures rejected by the $\sigma$-clipping algorithm.

\subsection{Measuring $\left\langle B_{z}\right\rangle$}

The mean longitudinal field $\left\langle B_{z}\right\rangle$ is obtained as explained in Sect. 3, using $V / I$ obtained via Eq. (13). Following common statistics guidelines, one should consider a detection as "definite" whenever the relation

$\left|\left\langle B_{z}\right\rangle\right| / \sigma\left[\left\langle B_{z}\right\rangle\right] \geq 3$

is satisfied.

We encountered a number of cases for which the field was detected at about 3- $\sigma$ level, and where minor changes in the data reduction would transform a marginal detection in a null or in a definite detection. Although these cases should certainly be investigated via additional observations, we tried to extract further information from the available spectra, to formulate a more robust and reliable criterion for field detection.

First, we decided to explore an alternative method for the determination of the mean longitudinal field. From the individual pairs of $V_{i j} / I_{i j}$ and $I_{i j}$ given by Eqs. (12), we calculated $m^{2}$ $\left\langle B_{z}\right\rangle_{i j}$ values, and the weighted mean longitudinal field

$\left\langle B_{z}\right\rangle^{\prime}=\sum_{i j}\left\langle B_{z}\right\rangle_{i j} \sigma^{-2}\left[\left\langle B_{z}\right\rangle_{i j}\right] / \sum_{i j} \sigma^{-2}\left[\left\langle B_{z}\right\rangle_{i j}\right]$.

The corresponding error bar $\sigma\left[\left\langle B_{z}\right\rangle^{\prime}\right]$ was calculated as

$\sigma^{2}\left[\left\langle B_{z}\right\rangle^{\prime}\right]=\frac{\sum_{i j=1}^{m}\left(\left\langle B_{z}\right\rangle_{i j}-\left\langle B_{z}\right\rangle^{\prime}\right)^{2}}{m^{2}(m-1)}$.
For each star, we systematically checked the consistency between the $\left\langle B_{z}\right\rangle$ value determined through the average $V / I$ obtained via Eq. (13) and the $\left\langle B_{z}\right\rangle^{\prime}$ value obtained via Eq. (17). We also checked whether the relation

$\left|\left\langle B_{z}\right\rangle^{\prime}\right| / \sigma\left[\left\langle B_{z}\right\rangle^{\prime}\right] \geq 3$

was satisfied.

Second, we performed a systematic analysis of metal lines. As pointed out earlier, Eq. (1) is formally valid only under the weak-field approximation. Therefore, in principle, $\left\langle B_{z}\right\rangle$ measurements by our method should be performed only on $\mathrm{H}$ Balmer lines. Furthermore, H Balmer lines are well sampled even at the low resolution of our observations, whereas metal lines are unresolved. Nevertheless, we found that the $\left\langle B_{z}\right\rangle$ determined via metal line analysis is consistent with that measured from $\mathrm{H}$ Balmer lines, provided that $\left\langle B_{z}\right\rangle$ is $\$ 800 \mathrm{G}$ (see Sect. 6.2). Therefore we decided to analyze the metal lines, i.e., to apply the least-squares technique to spectral regions free from $\mathrm{H}$ Balmer lines. In addition, we determined $\left\langle B_{z}\right\rangle$ using the whole spectrum, i.e., including both Balmer and metal lines. The outcome of this analysis will be discussed in Sect. 6 .

\section{Magnetic field determinations from Balmer lines vs. field determinations from metal lines}

\subsection{The magnetic "standard" star HD 94660}

In order to compare our results with those obtained through different techniques, we repeatedly observed a well known magnetic Ap star: HD 94660 (=KQ Vel). Previous $\left\langle B_{z}\right\rangle$ measurements of HD 94660 were obtained using the $\mathrm{H} \alpha$ Balmer line (Borra \& Landstreet 1975), the $\mathrm{H} \beta$ Balmer line (Bohlender et al. 1993), and metallic lines (Mathys 1994; Mathys \& Hubrig 1997). Moreover, HD 94660 is the star observed by Bagnulo et al. (2002) to develop the technique used in this work.

The log of our observations and $\left\langle B_{z}\right\rangle$ measurements is given in Table 1. Note that on 2002-02-04 we obtained two consecutive $\left\langle B_{z}\right\rangle$ measurements: the first one with grism $600 \mathrm{~B}$, the second one with grism $600 \mathrm{R}$.

Figure 2 shows the $\left\langle B_{z}\right\rangle$ measurements obtained from both the Balmer and the metal lines. The $\left\langle B_{z}\right\rangle$ values obtained from the Balmer lines blueward of $\mathrm{H} \beta$ are consistent among themselves, and marginally consistent with the $\left\langle B_{z}\right\rangle$ value obtained from $\mathrm{H} \alpha$. With the exception of the measurement taken on 2001-03-22, the $\left\langle B_{z}\right\rangle$ values obtained from the metal lines are not consistent with those obtained from Balmer lines. This is probably due to the fact that Eq. (1) is valid only under the 

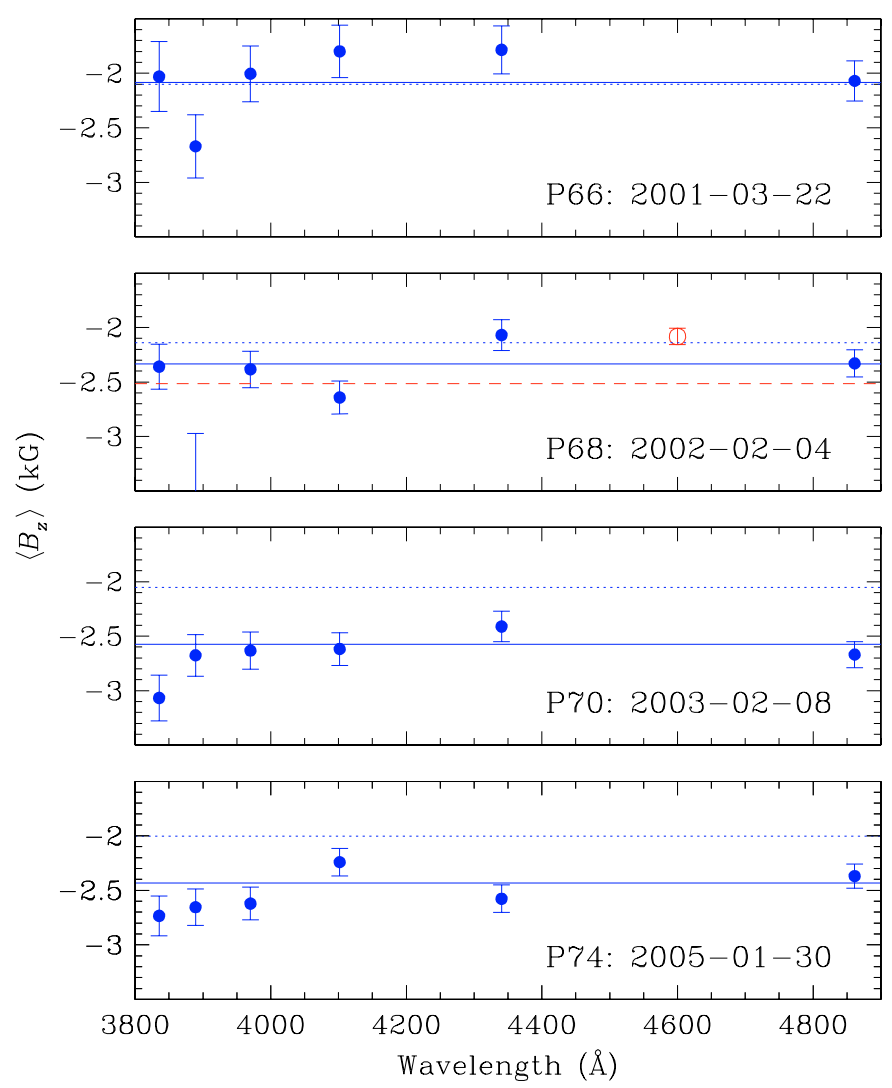

Fig. 2. $\left\langle B_{z}\right\rangle$ measurements of HD 94660 from individual Balmer lines. Each panel refers to a different observing date as specified in the figure. The $\left\langle B_{z}\right\rangle$ values measured from $\mathrm{H} \beta$ and blueward Balmer lines are plotted with filled circles at the corresponding wavelength. The $\left\langle B_{z}\right\rangle$ determination from $\mathrm{H} \alpha$ obtained on 2002-02-04 is arbitrarily plotted with an empty circle at $\lambda=4600 \AA$. The solid lines correspond to $\left\langle B_{z}\right\rangle$ obtained using all Balmer lines from $\mathrm{H} \beta$ down to the Balmer jump, and the dotted lines indicate $\left\langle B_{z}\right\rangle$ obtained from the metal lines with grism $600 \mathrm{~B}$. The dashed line in the second panel shows the $\left\langle B_{z}\right\rangle$ value obtained from the metal lines observed with grism $600 \mathrm{R}$.

weak field approximation (which in this case is correct for Balmer lines but not for metal lines). Note also that the $\left\langle B_{z}\right\rangle$ values measured via metal line analysis are not consistent among themselves if we compare data obtained with grism $600 \mathrm{~B}$ and grism $600 \mathrm{R}$. The comparison between $\left\langle B_{z}\right\rangle$ values obtained from $\mathrm{H}$ Balmer lines and metal lines will be further discussed in Sect. 6.2.

We also compared our $\left\langle B_{z}\right\rangle$ determinations of Table 1 with those previously obtained in the literature, adopting for the star's rotation period $2800 \mathrm{~d}$ (Landstreet \& Mathys 2000). The results are shown in Fig. 3. In general, it appears that $\left\langle B_{z}\right\rangle$ values obtained from Balmer lines are not consistent with the $\left\langle B_{z}\right\rangle$ determinations obtained using metallic lines. It seems likely that different methods used to evaluate $\left\langle B_{z}\right\rangle$ bear systematic differences (even though each may be internally consistent). Systematic inconsistencies between $\left\langle B_{z}\right\rangle$ determinations in Ap stars obtained with different chemical elements or with different techniques have been already found in previous works (see, for instance, Ryabchikova et al. 2005, who analysed several observations of HD 24712).

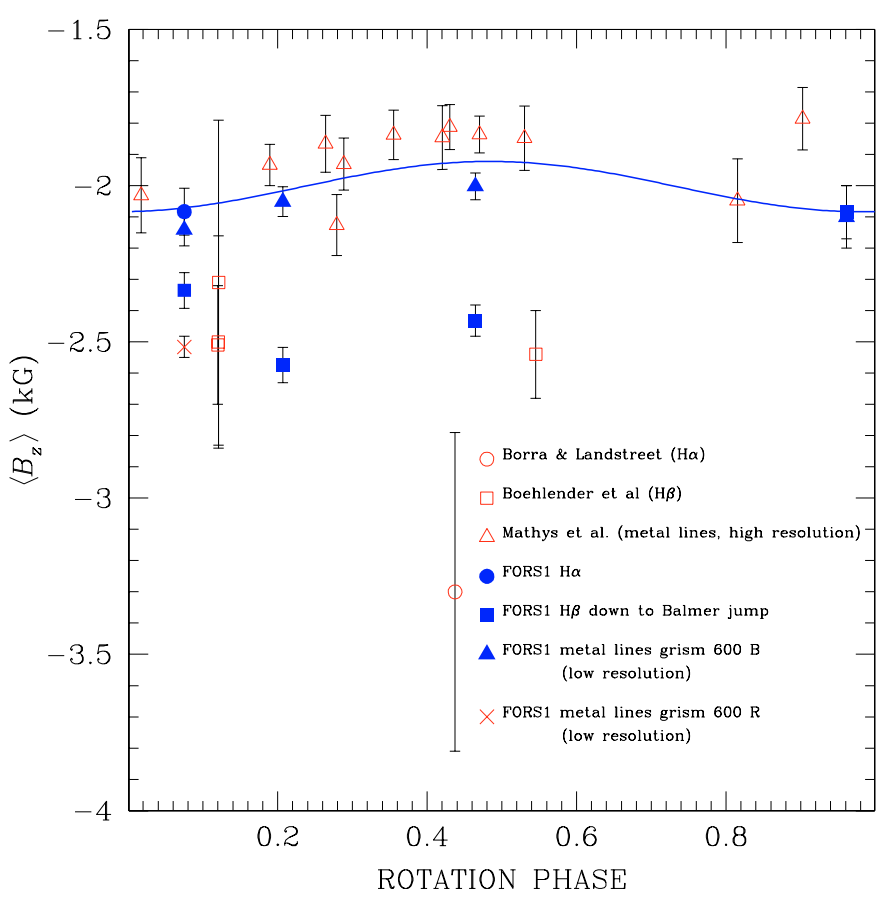

Fig. 3. Longitudinal field determinations of HD 94660. The solid line is a fit to the $\left\langle B_{z}\right\rangle$ determinations from metal lines. We have used a first-order Fourier expansion. At phase 0.075 we have considered the $\left\langle B_{z}\right\rangle$ determination obtained with grism $600 \mathrm{~B}$ and not the one obtained with grism $600 \mathrm{R}$. For the star's rotation period we adopt $2800 \mathrm{~d}$ (Landstreet \& Mathys 2000), and the zero phase point is at $\mathrm{JD}=2446500.0$.

\subsection{Weak field Ap stars}

Using all our data collected for Ap stars, we show here that the metal line analysis and the Balmer line analysis produce consistent results when the weak field hypothesis is satisfied.

Figure 4 shows the $\left\langle B_{z}\right\rangle$ values obtained from metal lines versus those obtained from Balmer lines for the observed Ap stars. It appears that the two methods give consistent $\left\langle B_{z}\right\rangle$ values for $\left|\left\langle B_{z}\right\rangle\right| \lesssim 800 \mathrm{G}$. Above the $1 \mathrm{kG}$ level, differences between Balmer line and metal line technique become noticeable or even striking. E.g., for HD 310187 we obtained from the Balmer lines $\left\langle B_{z}\right\rangle=6519 \pm 55 \mathrm{G}$, and from the metal lines we obtained $\left\langle B_{z}\right\rangle=3784 \pm 59 \mathrm{G}$. In general, above the $1 \mathrm{kG}$ level, the modulus of $\left\langle B_{z}\right\rangle$ determined from Balmer lines is larger than that from the metal lines, as we would expect from the earlier breakdown of the weak field expression for metal lines.

Summarizing, it appears that if the field is weak, both methods are consistent, and each gives an indication whether the star is magnetic or not independent of the other.

Figure 5 compares the distributions of the error bars for $\left\langle B_{z}\right\rangle$ obtained via Balmer and metal line analysis for the observed Ap stars. We see that the distribution of the error bars calculated via metal line analysis is broader than that obtained from Balmer lines. This is due primarily to the fact that Balmer lines have similar strength in all A and B-type stars, whereas metal lines may change greatly from star to star. For a given SNR, the error bars obtained via metal line analysis are smaller in spectra that are richer in metal lines than in spectra that are poorer in metal lines. As expected, for a set of observations 


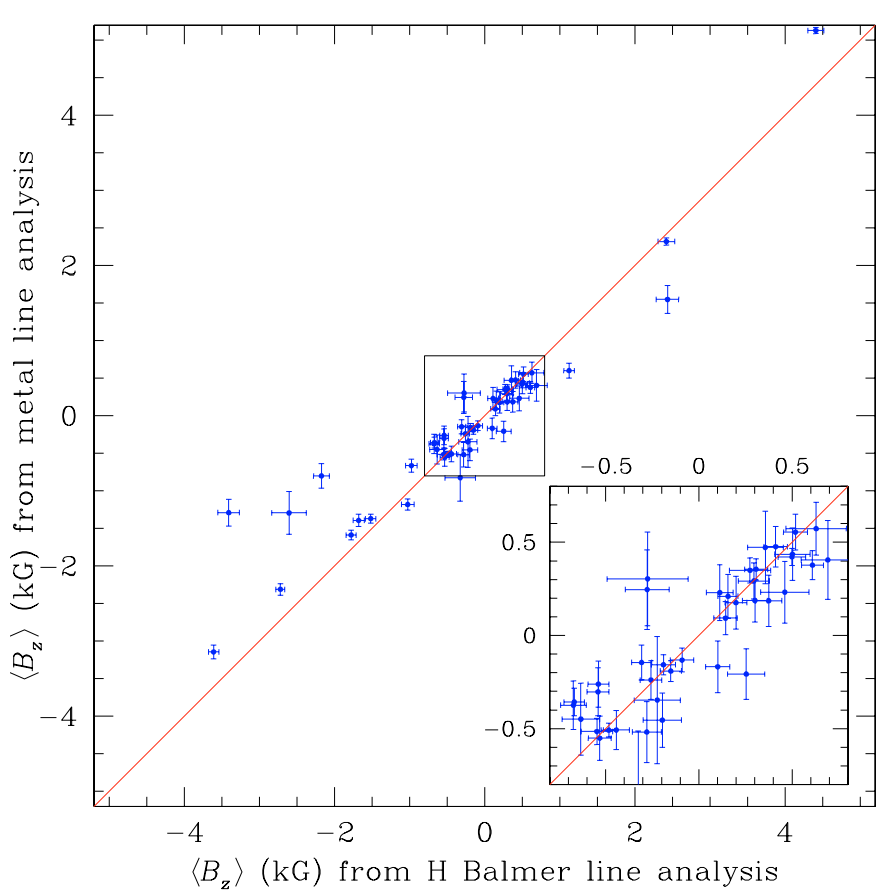

Fig. 4. $\left\langle B_{z}\right\rangle$ determinations from metal lines versus $\left\langle B_{z}\right\rangle$ determinations from Balmer lines for the stars of Table A.3. The solid line has slope $=1$. For clarity, only stars for which $\left\langle B_{z}\right\rangle$ obtained from Balmer lines is such that $\left|\left\langle B_{z}\right\rangle\right| / \sigma\left[\left\langle B_{z}\right\rangle\right] \geq 1$ have been plotted. The outliers HD 318107 and NGC 2244334 (see Table A.3) are not plotted.

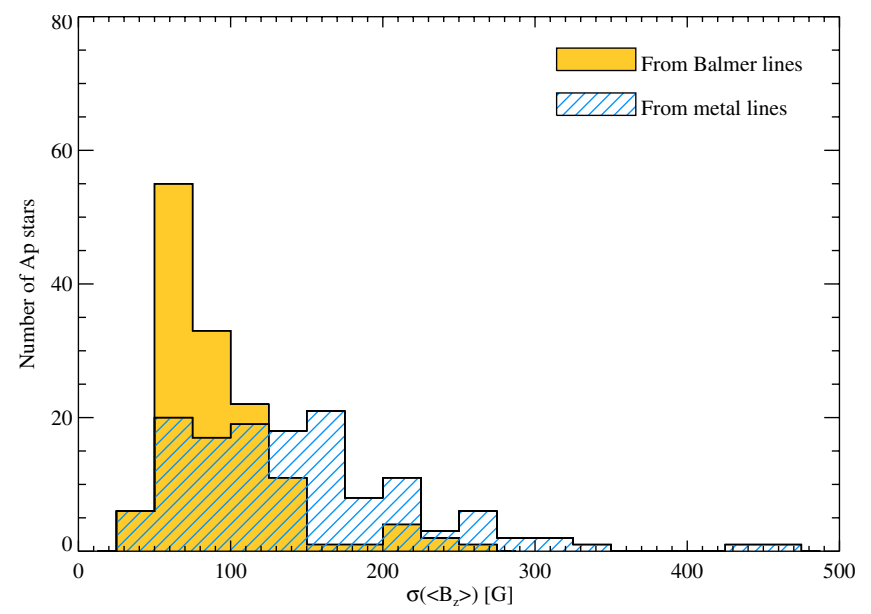

Fig. 5. Histograms of the error bars for the $\left\langle B_{z}\right\rangle$ measurements of Table A.3 obtained from Balmer lines and from metal lines. The median of the distribution of the error from Balmer lines is $78 \mathrm{G}$, from metal lines it is $130 \mathrm{G}$.

of similar SNR, the Balmer line analysis leads to results characterised by more homogeneous error bars than the metal line analysis.

\section{Observations}

In this survey we have observed 97 Ap stars, 138 normal A and B-type stars, and 22 non early-type stars. All these stars are candidate members of the open clusters (or the associations) listed in Table 2. Figure 6 gives an overview of the range in age and distance modulus of the target clusters, compared to
Table 2. List of open clusters observed in this survey, with approximate ages (Col. 4) and distance moduli $d=|M-m|$ (Col. 5) extracted from WEBDA. Column 6 gives the number of candidate peculiar stars and Col. 7 the number of non chemically peculiar A and B stars that we observed in each cluster.

\begin{tabular}{|c|c|c|c|c|c|c|}
\hline & & & & & & non \\
\hline RA \& & \& DEC & Name & $\log t$ & $d$ & Ap & Ap \\
\hline 000407 & -295000 & Blanco 1 & 7.796 & 7.18 & 1 & 0 \\
\hline 044827 & +105612 & NGC 1662 & 8.625 & 9.14 & 1 & 0 \\
\hline 0526 & +0305 & Ori OB1a ass. & 7.07 & 7.6 & 2 & 0 \\
\hline 0536 & -0112 & Ori OB1b ass. & 6.23 & 8.37 & 4 & 0 \\
\hline 0535 & -0450 & Ori OB1c ass. & 6.66 & 8.52 & 8 & 3 \\
\hline 0527 & -0523 & Ori OB1d ass. & 6.0 & 8.52 & 2 & 1 \\
\hline 060824 & +135754 & NGC 2169 & 7.067 & 10.73 & 1 & 4 \\
\hline 062801 & -045048 & NGC 2232 & 7.727 & 7.87 & 1 & 0 \\
\hline 063155 & +045630 & NGC 2244 & 6.896 & 12.23 & 1 & 3 \\
\hline 064601 & -204524 & NGC 2287 & 8.385 & 9.29 & 3 & 2 \\
\hline 065412 & -242500 & Collinder 121 & 7.054 & 8.45 & 1 & 0 \\
\hline 070242 & -082300 & NGC 2323 & 8.096 & 10.50 & 1 & 2 \\
\hline 070806 & -103700 & NGC 2343 & 7.104 & 10.48 & 0 & 28 \\
\hline 071520 & -304100 & Collinder 132 & 7.080 & 8.48 & 1 & 0 \\
\hline 073635 & -142900 & NGC 2422 & 7.861 & 8.67 & 4 & 2 \\
\hline 074312 & -382400 & NGC 2451a & 7.78 & 6.41 & 5 & 1 \\
\hline 075615 & -300348 & NGC 2489 & 7.264 & 14.15 & 2 & 1 \\
\hline 075804 & -604512 & NGC 2516 & 8.052 & 8.37 & 7 & 31 \\
\hline 081215 & -373542 & NGC 2546 & 7.874 & 10.23 & 5 & 5 \\
\hline 084032 & -530200 & IC 2391 & 7.661 & 6.24 & 2 & 0 \\
\hline 084754 & -472700 & Trumpler 10 & 7.542 & 8.24 & 1 & 0 \\
\hline 093311 & -532354 & NGC 2925 & 7.85 & 9.69 & 1 & 2 \\
\hline 100236 & -600712 & NGC 3114 & 8.093 & 10.01 & 6 & 4 \\
\hline 102122 & -542122 & NGC 3228 & 7.932 & 8.76 & 1 & 11 \\
\hline 103754 & -591100 & vdb-Hagen 99 & 7.605 & 8.70 & 1 & 1 \\
\hline 104258 & -642400 & IC 2602 & 7.507 & 6.11 & 1 & 0 \\
\hline 104204 & -595500 & Collinder 228 & 6.830 & 12.77 & 2 & 2 \\
\hline 110539 & -584512 & NGC 3532 & 8.492 & 8.55 & 3 & 3 \\
\hline 140727 & -482036 & NGC 5460 & 8.207 & 9.44 & 3 & 2 \\
\hline 143537 & -563706 & NGC 5662 & 7.968 & 10.08 & 2 & 5 \\
\hline 161850 & -575606 & NGC 6087 & 7.976 & 10.29 & 2 & 3 \\
\hline 163547 & -453836 & NGC 6178 & 7.248 & 10.71 & 2 & 0 \\
\hline 164120 & -484548 & NGC 6193 & 6.775 & 11.79 & 2 & 2 \\
\hline 170441 & -375906 & NGC 6281 & 8.497 & 8.86 & 2 & 1 \\
\hline 173448 & -323400 & NGC 6383 & 6.962 & 10.89 & 2 & 6 \\
\hline 174020 & -321512 & NGC 6405 & 7.974 & 8.88 & 4 & 9 \\
\hline 175351 & -344736 & NGC 6475 & 8.475 & 7.71 & 3 & 0 \\
\hline 182715 & +063030 & NGC 6633 & 8.629 & 8.44 & 2 & 0 \\
\hline 183147 & -190700 & IC 4725 & 7.965 & 10.44 & 4 & 4 \\
\hline 201719 & -790200 & Mel 227 & 8.57 & 5.52 & 1 & 0 \\
\hline TOTAL & & & & & 97 & 138 \\
\hline
\end{tabular}

all Milky Way open clusters with known age and $\delta \leq+10^{\circ}$. A summary of the observing log is given in Table 3.

Target Ap stars have been selected as explained in Sect. 2.3, and are listed in Table A.1, together with the $V$ magnitude and the spectral type extracted from the General Catalogue of Ap and Am stars (Renson et al. 1992), and from SIMBAD. Searching the literature (in particular the catalogue by 
Bychkov et al. 2003), we found that 10 of these Ap stars have already been checked for magnetic field in previous studies. We observed some of our objects more than once (we have made 121 observations of Ap stars). In total we obtained 52 field detections in 41 different stars (although for five stars we have only marginal detections). Thirty-seven Ap stars in which we have detected a field were not previously known as magnetic. A few Ap stars of Table A.1 are in fact cluster non-members. Chemical peculiarity, and, above all, cluster membership, will be further discussed in Paper II, where we will also provide estimates for star's temperature, mass, and evolutionary state. Two remarkable stars have been the subject of previous papers: HD 66318 (Bagnulo et al. 2003) and NGC 2244338 (Bagnulo et al. 2004).

The normal A and B-type stars observed in this survey are listed in Table A.2, together with their $V$ magnitudes and spectral classifications. In many cases the spectral type was not available in SIMBAD or in the literature, therefore, it has been determined through their color indices $B-V$ and $U-B$ extracted from WEBDA. The observed color indices have been corrected for extinction using the cluster $E(B-V)$ (also reported in WEBDA) and applying the extinction law by Cardelli et al. (1983). The dereddened colours have been then compared to those of typical main sequence stars to determine the approximate spectral type of each object. FORS1 spectra were used to roughly check the validity of this spectral classification, but no attempt was made to refine it. The spectral type obtained from the colour indices are reported between squared brackets, and should be used with caution. We discovered a posteriori that the spectra of $\sim 20$ normal stars were in fact not of early-type. These cases are listed in Table A.5. We do not report observations of a few additional targets which have spectra with a too low SNR to be useful for magnetic studies.

In this work we report the $\left\langle B_{z}\right\rangle$ values obtained from the analysis of both Balmer lines and metal lines. For peculiar stars, the medians of the errors obtained from the Balmer lines and from the metal lines are $78 \mathrm{G}$, and $130 \mathrm{G}$, respectively. For normal stars they are $136 \mathrm{G}$, and $173 \mathrm{G}$, respectively. For normal stars the median error bar is larger than for peculiar stars because we optimized integration times to reach the highest possible SNR for Ap stars, rather than for the secondary targets added in the fims mode observations. In addition, we calculated the field measured from the analysis of the whole spectrum, including metal lines. The latter method formally gives $\left\langle B_{z}\right\rangle$ values with the smallest error bar: for Ap stars the median is $62 \mathrm{G}$, and for normal stars is $94 \mathrm{G}$. Although some caution is required in interpreting a $\left\langle B_{z}\right\rangle$ determination obtained combining Balmer and metal lines, one can reasonably argue that this method may be used to reinforce the conclusions achieved with the two primary methods.

The $\left\langle B_{z}\right\rangle$ determinations for Ap stars and for normal A and B stars are reported in Tables A.3 and A.4, respectively. These tables are organized as follows. The day and time of the observations are given in Cols. 2, and 3, respectively, and correspond to the midpoint between the instant when the shutter opens for the first exposure and the time when the shutter closes after the last exposure. Columns 4-6 report the $\left\langle B_{z}\right\rangle$ values obtained from the analysis of the Balmer lines, the metal lines, and

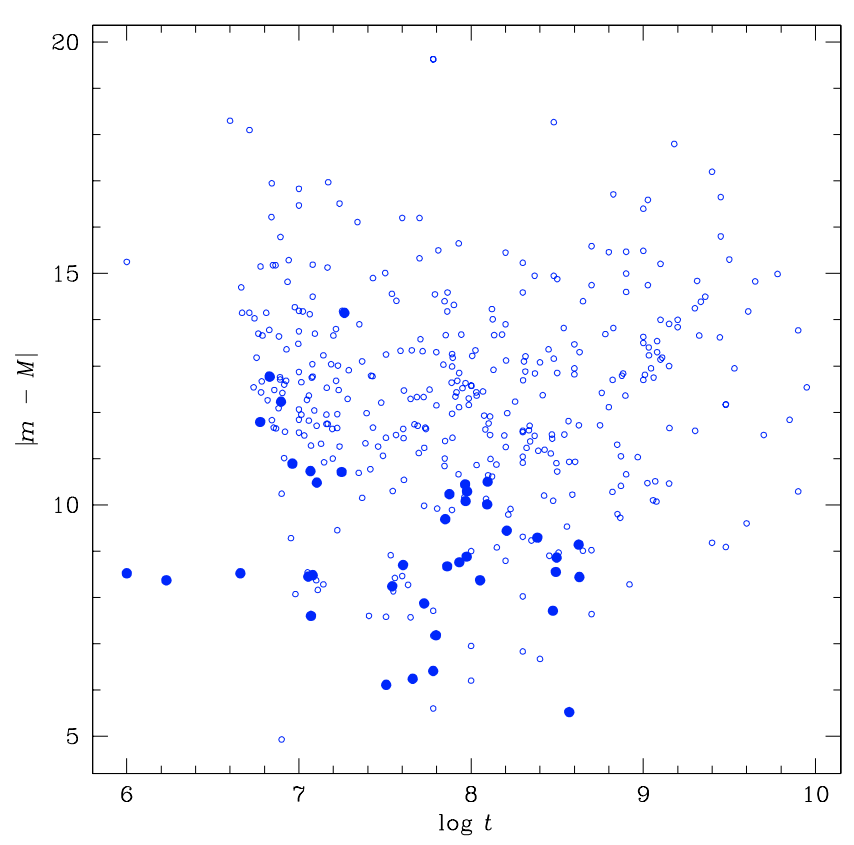

Fig. 6. Distance modulus versus age of open clusters. The empty circles show all open clusters with known age and $\delta<+10^{\circ}$. Filled circles are the clusters observed in this survey (same data as in Table 2). Data have been extracted from WEBDA.

the whole spectrum, respectively. All these $\left\langle B_{z}\right\rangle$ values are obtained through the least-squares technique applied to the averaged $V / I$ calculated via Eq. (13). For each method, we also systematically calculated $\left\langle B_{z}\right\rangle^{\prime}$ from Eq. (17). The cases in which both Eqs. (16) and (18) are satisfied are indicated in Col. 7 with symbol "D". The cases where only one of these equations is satisfied are flagged with symbol "d". If neither of Eqs. (16) or (18) is satisfied, we use the symbol "n". For example, in HD 62992, using the average spectrum obtained via Eq. (13), we have measured from Balmer lines $\left\langle B_{z}\right\rangle=-190 \pm 65 \mathrm{G}$. This value (slightly less than a $3-\sigma$ detection) is reported in col. 4 of Table A.3. From the average of the $\left\langle B_{z}\right\rangle$ values obtained from the individual spectra we obtain $\left\langle B_{z}\right\rangle^{\prime}=-186 \pm 54 \mathrm{G}$ (not reported in Table A.3). This is above a $3-\sigma$ detection, hence to this $\left\langle B_{z}\right\rangle$ determination we associate the symbol " $\mathrm{d}$ ".

This procedure is repeated for each method used (Balmer lines, metal lines, full spectrum), so that to each $\left\langle B_{z}\right\rangle$ value, a three-term flag is associated. This three-terms flag is meant to help to evaluate the significance of each field detection. A "DDD" sequence clearly indicates a firm field detection, and "nnn" sequence indicates a null detection, whereas intermediate cases deserve further investigation and discussion. In general, a "DnD" or "Dnd" sequence may still represent field detection, if the lack of a detection from the analysis of the metal lines can be explained by a metal spectrum with low line density. This is for instance the case of HD 35008 of Table A.3, where Balmer line analysis gives $\left\langle B_{z}\right\rangle=-340 \pm 72 \mathrm{G}$, and metal line analysis gives $\left\langle B_{z}\right\rangle=-273 \pm 293 \mathrm{G}$. Here note that metal line analysis does not confirm the magnetic field detection, but is still consistent with the field measured via Balmer line analysis. This star's spectrum is not rich in metal lines, explaining the large error bar of $\left\langle B_{z}\right\rangle$ obtained through the metal 
Table 3. Log of the various observing runs with FORS1 at the ESO VLT, dedicated to the open cluster survey.

\begin{tabular}{|c|c|c|c|c|c|}
\hline$\overline{\text { Period }}$ & Program ID & $\overline{\text { Dates }}$ & Observing Mode & $\overline{\text { Telescope }}$ & Grism \\
\hline P68 & 68.D-0403 & 2002 February $2 / 3$ and $3 / 4$ & Visitor & UT3 Melipal & $600 \mathrm{R}$ \\
\hline P70 & 70.D-0352 & 2003 February $7 / 8$ and $8 / 9$ & Visitor & UT1 Antu & $600 B \& 600 R$ \\
\hline P72 & 272.D-5026 & 2003 February $28 / 29$ to March $2 / 3$ & Service & UT1 Antu & $600 \mathrm{~B}$ \\
\hline P73 & 73.D-0498 & 2004 May $28 / 29$ to August $23 / 24$ & Service & UT1 Antu and UT2 Kueyen & $600 \mathrm{~B}$ \\
\hline P74 & 74.D-0488 & 2005 January $28 / 29$ and $29 / 30$ & Visitor & UT2 Kueyen & $600 \mathrm{~B}$ \\
\hline
\end{tabular}
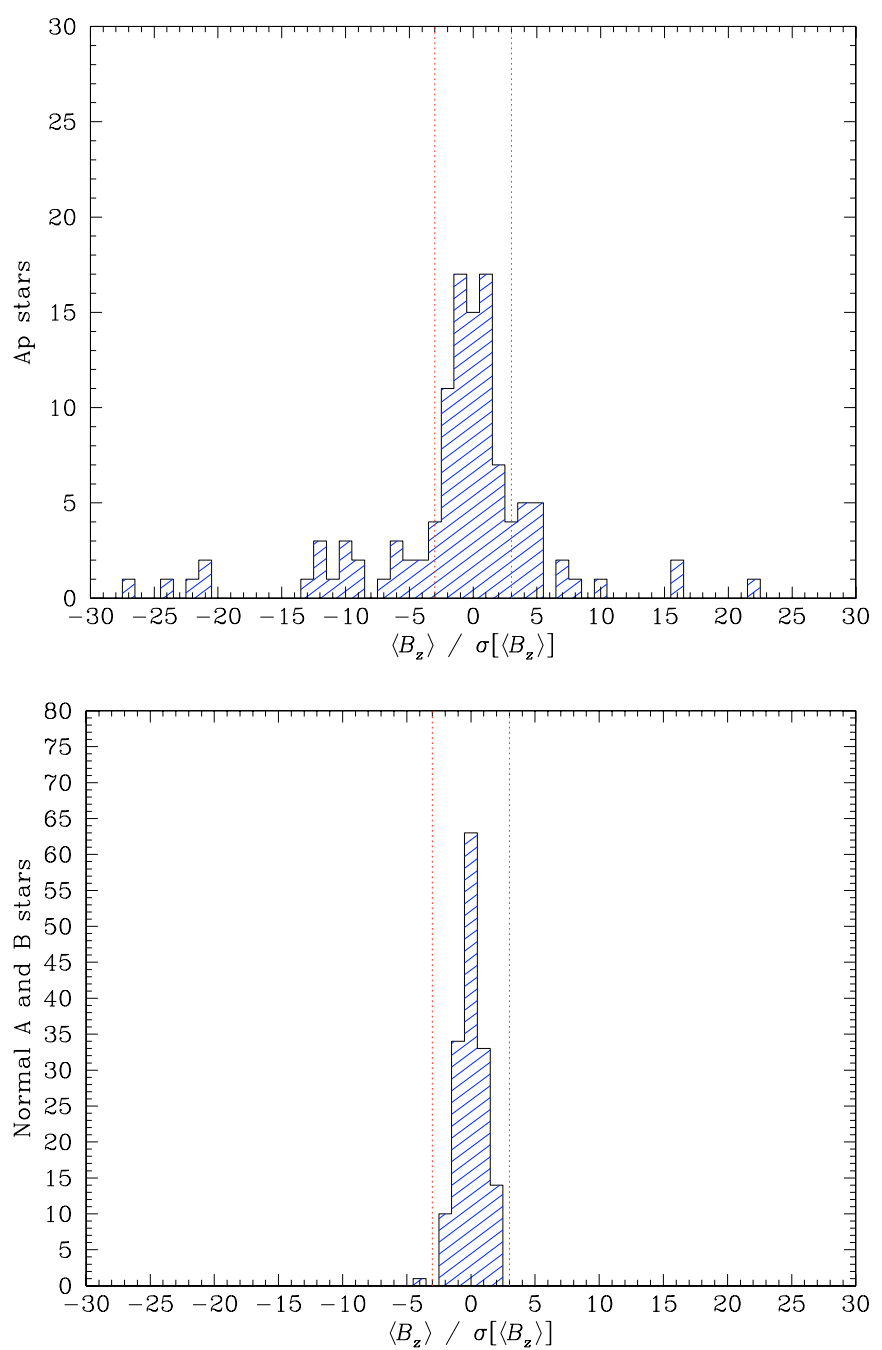

Fig. 7. Distribution of $\left\langle B_{z}\right\rangle$ values normalised to their error bars measured from Balmer lines in the Ap stars (upper panel) and in the normal A and B-type stars (lower panel). Five stars have been omitted from the upper panel (Ap stars) because they are off scale. The dotted lines correspond to the 3- $\sigma$ detection level.

line method. By contrast, a "Dnn", or a "dnn" sequence in a star with a large blocking factor would prompt a re-analysis of the data reduction, or defer judgment about field detection to further observations.

\section{Discussion}

In Fig. 7 we show the histograms of the $\left\langle B_{z}\right\rangle$ values calculated via Balmer line analysis and normalized to their error bars. The upper panel refer to the Ap stars, and the lower panel refers to the normal early-type stars. The top histogram shows that in $58 \%$ of the observed Ap stars we obtained a null detection. However, it would be incorrect to infer that only $\sim 40 \%$ of the Ap stars are magnetic. First, targets of Table A.1 have not been carefully checked for correct spectral classification, and some may not be chemically peculiar. Secondly, and most importantly, our magnetic field detection threshold is usually $\sim 250 \mathrm{G}$, whereas observations based on high resolution spectropolarimetry have shown that a number of Ap stars exhibit a smaller longitudinal field (e.g., Aurière et al. 2004). Finally, some of our targets may have been observed at rotation phases at which the longitudinal field is small. This appears to be the case for HD 74169, for which we detected a field in only one of two measurements. More detailed statistical considerations will be presented in Paper II.

Conversely, the bottom histogram of Fig. 7 shows that no star among the normal A and B-type shows evidence for magnetic field to a typical $3 \sigma$ detection limit of $400 \mathrm{G}$. This is fully consistent with the null detections obtained by Landstreet (1982) and by Shorlin et al. (2002) in their surveys based on $\mathrm{H} \beta$ photopolarimetry, and high resolution spectropolarimetry of metal lines, respectively. Landstreet (1982) observed 31 targets (including normal $\mathrm{A}$ and $\mathrm{B}$ stars, $\mathrm{Am}, \mathrm{HgMn}$ and $\lambda$ Bootis stars) providing measurements with a median error of $65 \mathrm{G}$. Shorlin et al. (2002) observed 64 targets with a typical error between 15 and $50 \mathrm{G}$. Our survey has a larger detection threshold, but is based on a larger sample. Therefore, our results bring substantial additional support to the common belief that Ap stars are the only objects of the middle main sequence characterized by strong, globally organized, magnetic fields.

Statistical data about rotation velocities show that the typical $v_{\mathrm{e}} \sin i$ values for normal $\mathrm{A}$ and B-type stars are in the range $100-200 \mathrm{~km} \mathrm{~s}^{-1}$, depending on the stellar mass. It is therefore reasonable to assume that our targets have $v_{\mathrm{e}} \sin i$ within the same range. Considering that observations based on high resolution spectropolarimetry are not suitable for field detection in rapidly rotating objects (say $v_{\mathrm{e}} \sin i \gtrsim 60 \mathrm{~km} \mathrm{~s}^{-1}$ ), our work represents the most extensive survey for magnetic fields in normal early-type stars, and is not biased toward slowly rotating stars. The null result of this survey suggests that magnetic field organized on large scales are not common in non-Ap stars. Finally, and most important, the null detection obtained in nonAp stars shows strong evidence that the diagnostic techniques used in this paper are not prone to spurious detections. The only exception is the star CD-60 1996, which deserves further investigation. Note however that with this many stars, statistically one should expect at least one measurement for which $\left|\left\langle B_{z}\right\rangle / \sigma\left[\left\langle B_{z}\right\rangle\right]\right|>3$, even in absence of any magnetic fields. 

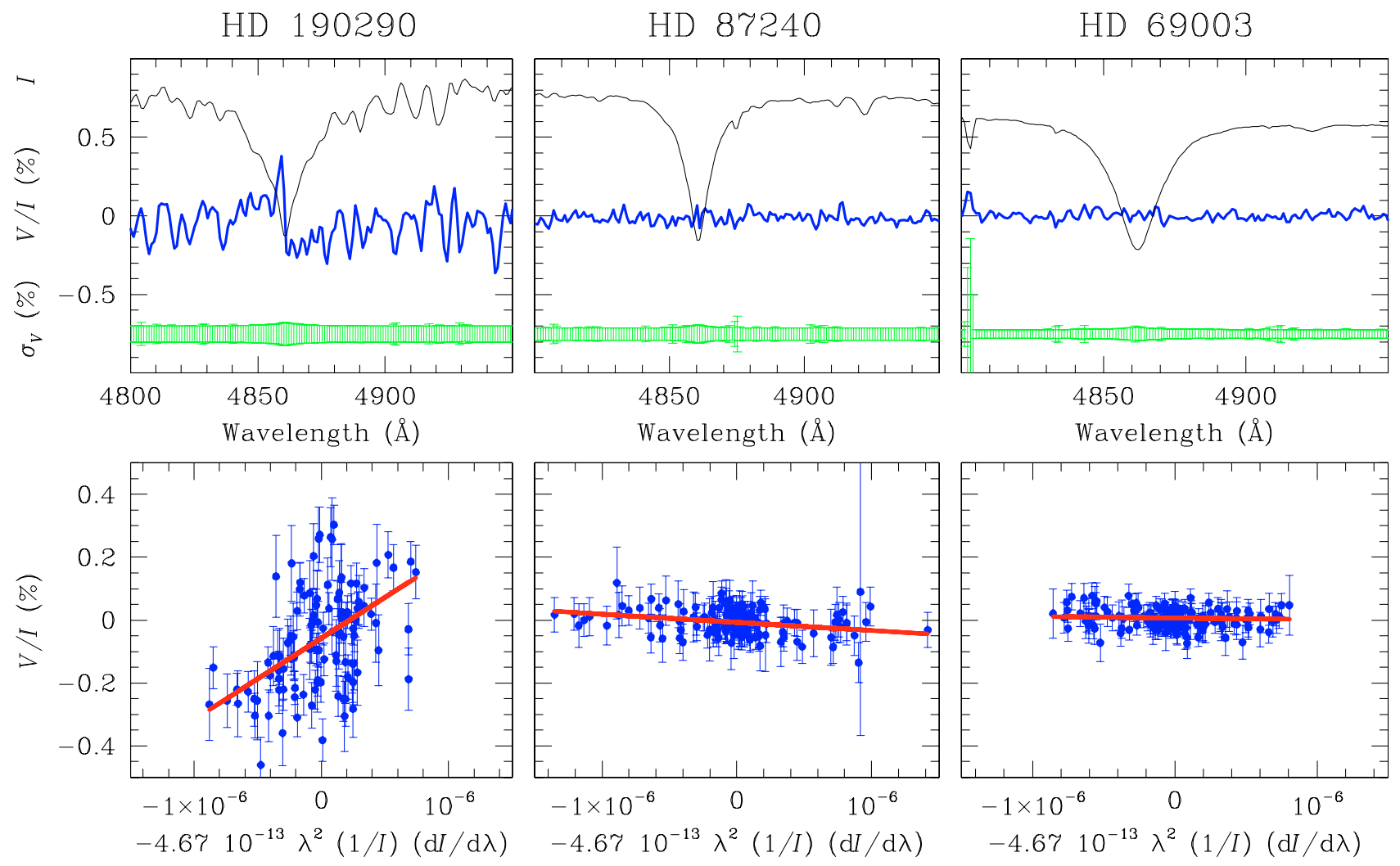

Fig. 8. Top panels: Stokes $I$ (thin solid lines, arbitrary units) $V / I$ profiles (thick solid line, in $\%$ ), and error bar associated to $V / I$, for three stars observed during the survey. Bottom panels: the corresponding best-fit to the Balmer lines obtained by minimizing the $\chi^{2}$ of Eq. (3). The longitudinal field measured in HD 190290 (left panels) is $\left\langle B_{z}\right\rangle=+2429 \pm 110 \mathrm{G}$; the field measured in HD 87240 (middle panels) is $-257 \pm 58 \mathrm{G}$; the field measured in HD 69003 (right panels) is $-48 \pm 53 \mathrm{G}$.

Examples of reduced data and $\left\langle B_{z}\right\rangle$ determinations for three selected stars are illustrated in Fig. 8, which shows $I$ and $V / I$ around $\mathrm{H} \beta$, as well as the best fit obtained applying Eq. (1) to all the Balmer lines. HD 190290 (left panels) exhibits a strong longitudinal field: $\left\langle B_{z}\right\rangle=+2429 \pm 110 \mathrm{G}$. HD 87240 (middle panels) has a magnetic field close to the detection limit: $\left\langle B_{z}\right\rangle=$ $-257 \pm 58$ G. HD 69003 (right panels) is a normal A-type star, and we did not detect a magnetic field $\left(\left\langle B_{z}\right\rangle=-48 \pm 53 \mathrm{G}\right)$. Note that a simple inspection to the $V / I$ profile of HD 87240 (middle panels) does not show any obvious polarization signal. The magnetic field is detected by the linear correlation between $V / I$ and the quantity $1 / I \times \mathrm{d} I / \mathrm{d} \lambda$.

Table A.5 reports a field detection in the star HD 298045 which is of spectral type M3. Although a field detection in a $\mathrm{M}$ giant would be an exciting discovery, this result must be taken very cautiously. The spectrum of the star is contaminated by a strong reflection from the LADC (see Sect. 5.1), and, although we have measured $\left\langle B_{z}\right\rangle$ using only those spectral regions that appear not contaminated, it is clear that the validity of our measurement is highly questionable. In any case, it would be certainly interesting to extend our technique also to late type stars.

\section{Conclusions}

We have carried out a survey of magnetic fields in A- and B-type stars belonging to open clusters and associations. The mean longitudinal magnetic field $\left\langle B_{z}\right\rangle$ has been measured in 257 stars, via the analysis of their circularly polarized spectra obtained with the FORS1 instrument of the ESO VLT. The results of this survey will be used, in Paper II, to study how magnetic fields of early-type stars change as stars evolve through the main sequence phase.

For the benefit of other FORS1 observers, we have discussed in depth the technical details of our strategy for observations, data reduction and data analysis. In particular, we have shown that a combined analysis of metal and $\mathrm{H}$ Balmer lines represents in many cases the best strategy to detect a weak stellar magnetic field.

Among the observed targets, 97 are candidate Ap cluster member stars. A magnetic field was detected in 41 Ap stars, 37 of which were not previously known as magnetic stars. In none of the remaining 160 non-peculiar stars have we detected a field. Among these, 138 are presumably normal A and B type stars, and 22 are late-type stars. The fact that no field was detected in any of the stars that are traditionally considered non magnetic convincingly shows that the techniques used in this paper are not prone to spurious detections. At the same time, this work represents one of the largest surveys for magnetic fields in normal A and B-type stars. The precision of this survey is not as high as that obtained through high resolution spectropolarimetry, but our survey is not biased toward slowly rotating stars. 
Before this work, only 13 candidate Ap cluster members, together with another $25 \mathrm{Ap}$ stars belonging to the Ori B and Sco-Cen association, had been searched for a magnetic field. Our survey has obtained observations for an additional $90 \mathrm{Ap}$ possible cluster members, and added about 30 more clusters to the list of those that have been searched for magnetic stars. For the first time we have now enough observational material to perform a preliminary search for links between magnetic field and evolution of Ap stars. This will be the subject of Paper II.

Acknowledgements. S.B., J.D.L., V.A., and G.A.W. are very grateful to W.W. Weiss for his kind hospitality at the Institut für Astronomie, Universität Wien. We wish to thank O. Kochukhov, F. Patat, and Th. Szeifert for useful discussions and suggestions. S.B. acknowledges DGDF 05/02 granted for a science leave in Wien. J.D.L. and G.A.W. acknowledge research support from the Natural Sciences and Engineering Research Council of Canada. JDL and VA acknowledge funding from the Scientific Visitors Programme of ESO Chile. This research has made use of the Simbad database, operated at CDS, Strasbourg, and of the WEBDA database, developed by J. Mermilliod and maintained at the Vienna Observatory by E. Paunzen.

\section{References}

Abt, H. A. 1979, ApJ, 230, 485

Aurière, M., Silvester, J., Wade, G. A., et al. 2004, in The A-star puzzle, ed. J. Zverko, J.Žižňovský, S. J. Adelman, \& W. W. Weiss (Cambridge University Press), IAUS, 224, 633

Babcock, H. W. 1947, ApJ, 105, 105

Babcock, H. W. 1958, ApJS, 3, 141

Bagnulo, S., Szeifert, T., Wade, G. A., Landstreet, J. D., \& Mathys, G. 2002, A\&A, 389, 191

Bagnulo, S., Landstreet, J. D., Lo Curto, G., Szeifert, T., \& Wade, G. A. 2003, A\&A, 403, 645

Bagnulo, S., Hensberge, H., Landstreet, J. D., Szeifert, T., \& Wade, G. A. 2004, A\&A, 416, 1149

Bevington, P. R. 1969, Data reduction and error analysis for the physical sciences (New York: McGraw-Hill)

Bohlender, D. A., Landstreet, J. D., \& Thompson, I. B. 1993, A\&A, 269,355

Bohlender, D. A., \& Landstreet, J. D. 1990, MNRAS, 247, 606

Borra, E. F. 1981, ApJ, 249, L39

Borra, E. F., \& Landstreet, J. D. 1975, PASP, 87, 961

Borra, E. F., \& Landstreet, J. D. 1980, ApJS, 42, 421

Borra, E. F., Landstreet, J. D., \& Thompson, I. 1983, ApJS, 53, 151

Bychkov, V. D., Bychkova, L. V., \& Madej, J. 2003, A\&A, 407, 631

Carrier, F., Burki, G., \& Richard, C. 1999, A\&A, 341, 469

Cardelli, J. A., Clayton, G. C., \& Mathis, J. S. 1989, ApJ, 345, 245

Casini, R., \& Landi Degl'Innocenti, E. 1994, A\&A, 291, 668

Castellani, V., Chieffi, A., \& Straniero, O. 1992, ApJS, 78, 517

Chen, L., Hou, J. L., \& Wang, J. J. 2003, AJ, 125, 1397

Conti, P. S. 1970, ApJ, 159, 723

Cowley, C. R., \& Bord, D. J. 2004, in The A-Star Puzzle, ed. J.Zverko, J.Žižňovský, S. J. Adelman, \& W. W. Weiss (Cambridge University Press), IAUS, 224, 265

de Zeeuw, P. T., Hoogerwerf, R., de Bruijne, J. H. J., Brown, A. G. A., \& Blaauw, A. 1999, ApJ, 117, 354

Dachs, J., \& Kabus, H. 1989, A\&AS, 78, 25

Dias, W. S., Lépine, J. R. D., \& Alessi, B. S. 2001, A\&A, 376, 441
Donati, J.-F., Semel, M., Carter, B. D., et al. 1997, MNRAS, 291, 658 Elkin, V. G., Kudryavtsev, D. O., \& Romanyuk, I. I. 2003, AstL, 29, 400

Gomez, A. E., Luri, X., Grenier, S., et al. 1998, A\&A, 336, 953

Joncas, G., \& Borra, E. F. 1981, A\&A, 94, 134

Hartoog, M. R. 1976, ApJ, 205, 807

Hartoog, M. R. 1977, ApJ, 212, 723

Hauck, B., \& North, P. 1982, A\&A, 114, 23

Hauck, B., \& North, P. 1993, A\&A, 269, 403

Høg, E., Kuzmin, A., Bastian, U., et al. 1998, A\&A, 335, L65

Høg, E., Fabricius, C., Makarov, V. V., et al. 2000, A\&A, 355, L27

Houk, N., \& Smith-Moore, M. 1988, Michigan Spectral Survey, Ann Arbor, Dept. of Astronomy, Univ. of Michigan, Vol. 4

Huber, P. J. 1981, Robust Statistics, ed. J. Wiley, \& Sons, New York

Hubrig, S., North, P., \& Mathys, G. 2000, ApJ, 539, 352

Hubrig, S., Szeifert, T., Schöller, M., Mathys, G., \& Kurtz, D. W. 2004, A\&A, 415, 685

Kochukhov, O., \& Bagnulo, S. 2006, A\&A, in press [arXiv: astro-ph/0601461]

Kramer, N., \& Maeder, A. 1980, A\&A, 88, 135

Kupka, F., Paunzen, E., \& Maitzen, H. M. 2003, MNRAS, 341, 849

Landstreet, J. D. 1982, ApJ, 258, 639

Landstreet, J. D., \& Mathys, G. 2000, A\&A, 359, 213

Lanz, T. 1984, A\&A, 139, 161

Maitzen, H. M. 1993, A\&AS, 102, 1

Mathys, G. 1991, A\&AS, 89, 121

Mathys, G. 1994, A\&AS, 108, 547

Mathys, G. 2004, in The A-Star Puzzle, ed. J.Zverko, J.Žižňovský, S. J. Adelman, \& W. W. Weiss (Cambridge University Press), IAUS, 224, 225

Mathys, G., \& Hubrig, S. 1997, A\&AS, 124, 475

Mathys, G., Hubrig, S., Landstreet, J. D., Lanz, T., \& Manfroid, J. 1997, A\&AS, 123, 353

Mermilliod, J.-C., \& Paunzen, E. 2003, A\&A, 410, 511

Napiwotzki, R., Schönberner, D., \& Wenske, V. 1993, A\&A, 268, 653

Pöhnl, H., Paunzen, E., \& Maitzen, H. M. 2005, A\&A, 441, 1111

Preston, G.W., \& Stępień, K. 1968, ApJ, 151, 577

Renson, P. 1992, Bull. Inf. Centre Donnees Stellaires, 40, 97

Renson, P., Gerbaldi, M., \& Catalano, F. A. 1991, A\&AS, 89, 429

Robichon, N., Arenou, F., Mermilliod, J.-C., \& Turon, C. 1999, A\&A, 345,471

Ryabchikova, T., Wade, G. A., Aurière, M., et al. 2005, A\&A, 429, 55

Sargent, W. L. W., Sargent, A. I., \& Strittmatter, P. A. 1967, ApJ, 147, 1185

Schaerer, D., Meynet, G., Maeder, A., \& Schaller, G. 1993, A\&AS, 98, 523

Schaller, G., Schaerer, D., Meynet, G., \& Maeder, A. 1992, A\&ASS, 96, 269

Seifert, W., Appenzeller, I., Fürtig, W., et al. 2000, SPIE, 4008, 96

Shorlin, S. L. S., Wade, G. A., Donati, J.-F., et al. 2002, A\&A, 392, 637

Sokolov, N. A. 1998, Contributions of the Astronomical Observatory Skalnate Pleso, 27, 261

Stẹpień, K. 2000, A\&A, 353, 227

Stȩpień, K., \& Dominiczak, R. 1989, A\&A, 219, 197

Thompson, I. B., Brown, D. N., \& Landstreet, J. D. 1987, ApJS, 64, 219

Wade, G. A., Donati, J.-F., Landstreet, J. D., \& Shorlin, S. L. S. 2000, MNRAS, 313, 851 
S. Bagnulo et al.: A survey of magnetic fields in open cluster A- and B-type stars with FORS1. I., Online Material p 1

\section{Online Material}




\section{Appendix A: The standard deviation of $\overline{V / I}$}

We begin by defining, for brevity and clarity, the following quantities:

$$
\begin{aligned}
v & \equiv \frac{V}{I}, \\
\phi^{ \pm} & \equiv\left(\frac{f^{\mathrm{o}}-f^{\mathrm{e}}}{f^{\mathrm{o}}+f^{\mathrm{e}}}\right)_{\alpha= \pm 45^{\circ}} .
\end{aligned}
$$

With these definitions, Eq. (7) becomes:

$v=\phi^{-}-\phi^{+}$.

We now consider a sequence of $n$ exposures taken with $\alpha=$ $-45^{\circ}$, and $m$ exposures taken with $\alpha=+45^{\circ}$, thus obtaining a set of $K=n m$ pairs, $v_{i j}=\phi_{i}^{-}-\phi_{j}^{+}$. In general we have that $m \neq n$, if for instance one or more data point have been rejected by the $\sigma$-clipping algorithm described in Sect. 5.3.

From such a set of measurements, we estimate the expected value for $v$ using Eq. (13), that is:

$$
\bar{v} \equiv \frac{\sum_{i j} v_{i j} / \sigma_{i j}^{2}}{\sum_{i j} 1 / \sigma_{i j}^{2}},
$$

where we have shortened the notation for $\sigma^{2}\left[v_{i j}\right]=\sigma^{2}\left[\phi_{i}^{-}\right]+$ $\sigma^{2}\left[\phi_{j}^{+}\right]$with $\sigma_{i j}^{2}$.

We now wish to estimate the variance of the statistical variable $\bar{v}$ :

$$
\begin{aligned}
\sigma^{2}[\bar{v}] & =\left\langle(\bar{v}-\langle v\rangle)^{2}\right\rangle=\left(\sum_{i=1}^{n} \sum_{j=1}^{m} \sigma_{i j}^{-2}\right)^{-2} \\
\times & \left(\sum_{i, h=1}^{n} \sum_{j, k=1}^{m} \sigma_{i j}^{-2} \sigma_{h k}^{-2}\left\langle\left(\phi_{i}^{-}-\phi_{j}^{+}-\langle v\rangle\right)\left(\phi_{h}^{-}-\phi_{k}^{+}-\langle v\rangle\right)\right\rangle,\right.
\end{aligned}
$$

where $\langle v\rangle$ is of course the mean value of $v$ and of $\bar{v}$. Since, obviously, $\langle v\rangle=\left\langle\phi^{-}\right\rangle-\left\langle\phi^{+}\right\rangle$(from Eq. (A.3)), and since the various exposures are statistically uncorrelated (thus the covariance $\left.\sigma\left[\phi^{-}, \phi^{+}\right]=0\right)$, it is easy to derive:

$$
\begin{aligned}
\sigma^{2}[\bar{v}] & =\left(\sum_{i=1}^{n} \sum_{j=1}^{m} \sigma_{i j}^{-2}\right)^{-2} \\
\times & {\left[\sum_{i=1}^{n} \sum_{j=1}^{m} \sigma_{i j}^{-2}\left(\sigma^{2}\left[\phi_{i}^{-}\right] \sum_{k=1}^{m} \sigma_{i k}^{-2}+\sigma^{2}\left[\phi_{j}^{+}\right] \sum_{h=1}^{n} \sigma_{h j}^{-2}\right)\right] . }
\end{aligned}
$$

If all errors of individual exposures can be assumed equal:

$\sigma^{2}\left[\phi_{i}^{-}\right]=\sigma^{2}\left[\phi_{j}^{+}\right]=\sigma_{i j}^{2} / 2=\sigma^{2} / 2$,

for $i=1, \ldots n$ and $j=1, \ldots m$, Eqs. (A.4) and (A.5) become, respectively:

$$
\begin{aligned}
\bar{v} & =\frac{1}{n m} \sum_{i j} v_{i j}=\overline{\phi^{-}}-\overline{\phi^{+}} \\
\sigma^{2}[\bar{v}] & =\frac{\sigma^{2}}{2}\left(\frac{1}{n}+\frac{1}{m}\right) .
\end{aligned}
$$

The latter equation, in particular, reduces to Eq. (14) if $n=m$. In this case, it is worth noting that at the denominator of
Eq. (14) there appears $m$ instead of the number of exposure pairs $K=m^{2}$, as it would naively be expected from the analogy with the average of $m^{2}$ independent measures. The reason is obviously that the $m^{2}$ combinations are not all completely independent from each other, since each exposure is counted $m$ times in Eqs. (13) and (A.4).

It would be desirable to find an alternative to Eq. (A.5) that does not depend explicitly on the knowledge of the statistical errors of the individual exposures. We seek an estimator of the form:

$s^{2}=C \sum_{i j}\left(v_{i j}-\bar{v}\right)^{2}$,

where $C$ is a constant such that the statistical estimator $s^{2}$ is not biased, i. e. its mean value coincides with the expected value $\sigma^{2}[\bar{v}]$. We find such a constant under the assumption that all errors of individual exposures are assumed equal (Eq. A.6):

$$
\begin{aligned}
\left\langle s^{2}\right\rangle & =C\left\langle\sum_{i j}\left(v_{i j}-\bar{v}\right)^{2}\right\rangle \\
& =C \sum_{i j}\left\langle\left[\left(v_{i j}-\langle v\rangle\right)-(\bar{v}-\langle v\rangle)\right]^{2}\right\rangle \\
& =C\left[n m\left(\sigma^{2}[v]+\sigma^{2}[\bar{v}]\right)-2 n m\left\langle(\bar{v}-\langle v\rangle)^{2}\right\rangle\right] \\
& =C\left[n m\left(\sigma^{2}[v]-\sigma^{2}[\bar{v}]\right)\right] \\
& =C[m(n-1)+n(m-1)] \sigma^{2} / 2,
\end{aligned}
$$

where in the last passage we have used Eq. (A.8). From the above expressions, the estimator $s^{2}$ is unbiased if it is defined as:

$s^{2}=\frac{1}{m n[2 n m /(n+m)-1]} \sum_{i j}\left(v_{i j}-\bar{v}\right)^{2}$,

which, of course, becomes Eq. (15) if $m=n$. 
S. Bagnulo et al.: A survey of magnetic fields in open cluster A- and B-type stars with FORS1. I., Online Material p 3

Table A.1. List of Ap star candidate open cluster members. Magnitude and spectral types are taken from the General catalogue of Ap and Am stars by Renson et al. (1992), and/or from SIMBAD. In Col. 6 we report a flag about peculiarity confidence from the same catalogue. Symbol * means "well knonw Ap star", symbol "?" means "doubtful nature"; we indicate with "-" the cases in which the star is not included in the Renson et al's catalogue (in which case spectral type is taken from SIMBAD or specific literature). The star's actual membership and peculiarity will be discussed in Paper II.

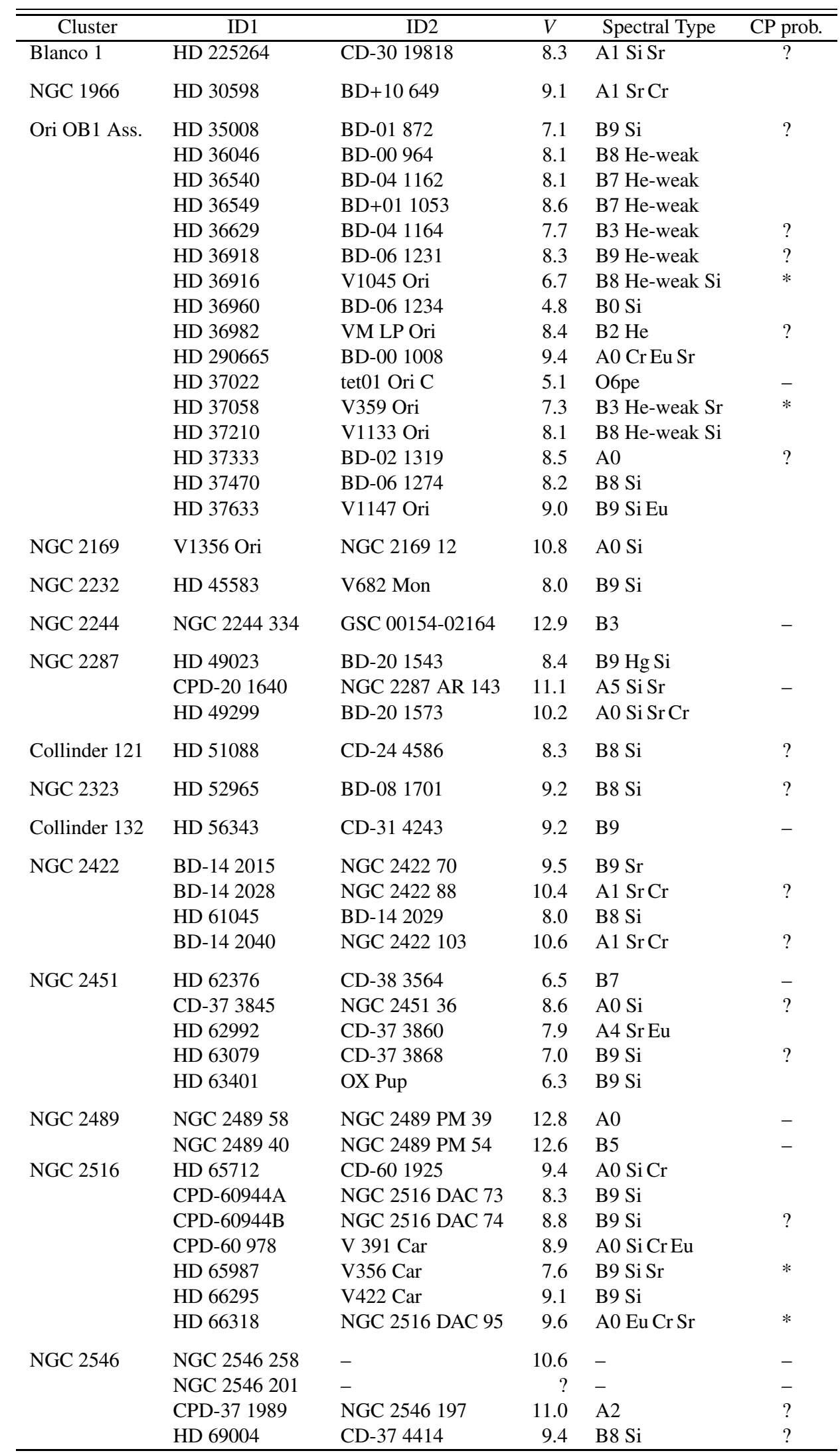


S. Bagnulo et al.: A survey of magnetic fields in open cluster A- and B-type stars with FORS1. I., Online Material p 4

Table A.1. continued.

\begin{tabular}{|c|c|c|c|c|c|}
\hline Cluster & $\overline{\mathrm{ID} 1}$ & ID2 & $\bar{V}$ & Spectral Type & CP prob. \\
\hline & HD 69067 & CD-37 4420 & 8.3 & B8 Si & \\
\hline \multirow[t]{2}{*}{ IC 2391} & HD 74169 & KR Vel & 7.2 & $\mathrm{~A} 1 \mathrm{SiCrSr}$ & $*$ \\
\hline & HD 74535 & KT Vel & 5.6 & B9 Si & $*$ \\
\hline Trumpler 10 & HD 75239 & CD-41 4498 & 9.2 & B9 $\mathrm{Si}$ & \\
\hline NGC 2925 & HD 83002 & CD-52 3172 & 9.1 & B8 Si & \\
\hline \multirow{6}{*}{ NGC 3114} & HD 87241 & CD-59 2746 & 7.8 & B9 Si & $?$ \\
\hline & HD 87240 & CPD-59 1673 & 9.6 & B9 Si & \\
\hline & HD 87266 & CD-59 2755 & 8.2 & B3 Si N & \\
\hline & HD 304841 & CPD-59 1717 & 10.0 & A & $?$ \\
\hline & HD 304842 & V424 Car & 9.7 & B8 Si & \\
\hline & HD 87405 & CD-59 2803 & 8.5 & B9 Si & \\
\hline NGC 3228 & HD 89856 & CD-51 4685 & 9.1 & B8 & $?$ \\
\hline vdB-Hagen88 & HD 92190 & CD-58 3407 & 8.6 & B8 & - \\
\hline IC 2602 & HD 92385 & V 407 Car & 6.7 & B9 Si & \\
\hline \multirow[t]{2}{*}{ Collinder 228} & Collinder 22830 & Collinder 228 CP4 & 10.8 & B1 & - \\
\hline & HD 305451 & CPD-59 2496 & 10.5 & B9 Si & $?$ \\
\hline \multirow[t]{3}{*}{ NGC 3532} & HD 96040 & CPD-57 4168 & 10.7 & A & $?$ \\
\hline & HD 96729 & CPD-58 3157 & 10.0 & B9 Si & \\
\hline & HD 303821 & NGC 3532241 & 11.7 & A & $?$ \\
\hline \multirow[t]{3}{*}{ NGC 5460} & HD 122983 & CD-47 8870 & 9.9 & A0 & $?$ \\
\hline & HD 123183 & CD-47 8895 & 9.9 & A0 & - \\
\hline & HD 123225 & CD-47 8901 & 8.9 & B9 $\mathrm{Si}$ & $?$ \\
\hline \multirow[t]{2}{*}{ NGC 5662} & CPD-56 6330 & NGC 566285 & 10.6 & $\mathrm{~A} 2$ & \\
\hline & HD 127866 & CD-56 5516 & 8.3 & B8 & - \\
\hline \multirow[t]{2}{*}{ NGC 6087} & CPD-57 7817 & NGC 608725 & 10.0 & - & $?$ \\
\hline & HD 146555 & CPD-57 7872 & 10.3 & B9 Si & \\
\hline \multirow[t]{2}{*}{ NGC 6178} & HD 149257 & CD-45 10768 & 8.5 & B5 He & \\
\hline & HD 149277 & CD-45 10769 & 8.4 & B2 & - \\
\hline \multirow[t]{2}{*}{ NGC 6193} & CD-48 11051 & CPD-48 8684 & 10.4 & $\mathrm{~B} 1$ & - \\
\hline & CD-48 11059 & NGC 6193 VF 33 & 10.7 & B3 & - \\
\hline \multirow[t]{2}{*}{ NGC 6281} & HD 322676 & CD-37 11203 & 10.2 & A0 & - \\
\hline & HD 153948 & V948 Sco & 9.3 & $\mathrm{~A} 2 \mathrm{Si}$ & \\
\hline \multirow[t]{2}{*}{ NGC 6383} & NGC 638326 & NGC 6383 FJL 5 & 12.9 & A & - \\
\hline & HD 317857 & NGC 63833 & 10.3 & A2 & - \\
\hline \multirow[t]{4}{*}{ NGC 6405} & HD 318107 & V970 Sco & 9.3 & B8 & $?$ \\
\hline & HD 318100 & V971 Sco & 9.8 & B9 & \\
\hline & CD-32 13119 & NGC 64057 & 10.9 & - & $?$ \\
\hline & HD 318095 & - & 10.9 & A0 & $?$ \\
\hline \multirow[t]{3}{*}{ NGC 6475} & HD 162305 & CD-34 12154 & 7.8 & B9 & $?$ \\
\hline & HD 320764 & CD-34 12161 & 8.9 & A6 & $?$ \\
\hline & HD 162725 & V951 Sco & 6.4 & B9 $\mathrm{SiCr}$ & $*$ \\
\hline \multirow[t]{2}{*}{ NGC 6633} & HD 169959 & $\mathrm{BD}+063762$ & 7.8 & $\mathrm{~A} 0 \mathrm{Si}$ & \\
\hline & HD 170054 & $\mathrm{BD}+063772$ & 8.2 & B7 Si & \\
\hline \multirow[t]{4}{*}{ IC 4725} & BD-19 5044L & CPD-19 6897 & 10.2 & B8 & $?$ \\
\hline & BD-19 5046 & CPD-19 6905 & 9.0 & A1 & - \\
\hline & HD 170836 & BD-19 5052 & 9.0 & B7 & $?$ \\
\hline & HD 170860A & BD-19 5058 & 9.4 & B8 & - \\
\hline Melotte 227 & HD 190290 & CK Oct & 9.9 & $\mathrm{~A} 0 \mathrm{Eu} \mathrm{Sr}$ & \\
\hline
\end{tabular}


S. Bagnulo et al.: A survey of magnetic fields in open cluster A- and B-type stars with FORS1. I., Online Material p 5

Table A.2. List of the observed normal cluster A and B-type stars. Actual membership has not been checked. Spectral types reported between square brackets were estimated from the star's colour indices, and should be taken with caution.

\begin{tabular}{|c|c|c|c|c|}
\hline$\overline{\text { Cluster }}$ & $\overline{\overline{\text { ID1 }}}$ & $\overline{\mathrm{ID} 2}$ & $\overline{\bar{V}}$ & $\overline{\text { Spectral Type }}$ \\
\hline \multirow[t]{4}{*}{ Ori OB1 Ass. } & HD 36559 & BD-04 1163 & 8.8 & B9 \\
\hline & HD 36671 & BD-04 1165 & 8.7 & B9 \\
\hline & HD 37041 & tet02 Ori $\mathrm{A}$ & 5.1 & O9.5Vpe \\
\hline & HD 37428 & BD-06 1271 & 8.8 & A0 \\
\hline \multirow[t]{4}{*}{ NGC 2169} & HD 252214 & $\mathrm{BD}+131120$ & 8.1 & $\mathrm{~B} 2.5 \mathrm{~V}$ \\
\hline & HD 41909 & $\mathrm{BD}+141160$ & 8.4 & B5 \\
\hline & HD 252248 & $\mathrm{BD}+131123$ & 8.8 & $\mathrm{~B} 2 \mathrm{~V}$ \\
\hline & HD 252266 & $\mathrm{BD}+131124 \mathrm{E}$ & 9.2 & $\mathrm{~B} 3 \mathrm{~V}$ \\
\hline \multirow[t]{3}{*}{ NGC 2244} & NGC 2244336 & GSC 00154-01288 & 12.6 & $\mathrm{~A} 0$ \\
\hline & CPD-20 1637 & NGC 228754 & 10.9 & A1V \\
\hline & BD-20 1571 & NGC 228755 & 10.8 & A3 \\
\hline \multirow[t]{2}{*}{ NGC 2287} & NGC 2287 AR 157 & - & $?$ & $?$ \\
\hline & CPD-20 1645 & NGC 2287 AR 159 & $?$ & $?$ \\
\hline \multirow[t]{2}{*}{ NGC 2323} & HD 52980 & BD-08 1796 & 8.4 & B9 \\
\hline & BD-08 1708 & NGC 232351 & 9.9 & B6 \\
\hline \multirow[t]{28}{*}{ NGC 2343} & CSI-10-07049 & NGC 234313 & 10.9 & - \\
\hline & BD-10 1875 & NGC 23439 & 10.6 & [A0] \\
\hline & NGC 234322 & CSI-10-07051 & 11.8 & {$[\mathrm{~A} 0 \mathrm{~V}]$} \\
\hline & NGC 234340 & CSI-10-07053 3 & 13.1 & {$[\mathrm{~F} 1 ? \mathrm{~V}]$} \\
\hline & CSI-10-07053 5 & NGC 234335 & 12.9 & {$[\mathrm{~A} 6 \mathrm{~V}]$} \\
\hline & BD-10 1878 & NGC 234312 & 10.8 & {$[\mathrm{~B} 8 / 9]$} \\
\hline & NGC 234325 & CSI-10-07054 1 & 11.7 & {$[\mathrm{~A} 3 / 4 \mathrm{~V}]$} \\
\hline & NGC 234316 & CSI-10-07055 1 & 11.4 & [A8?] \\
\hline & BD-10 1879a & NGC 23437 & 10.3 & {$[\mathrm{~A} 3 \mathrm{~V}]$} \\
\hline & NGC 234334 & CSI-10-07056 5 & 12.7 & {$[\mathrm{~A} 6 \mathrm{~V}]$} \\
\hline & NGC 234343 & CSI-10-07056 6 & 13.6 & [F?] \\
\hline & NGC 234328 & CSI-10-07055 2 & 12.5 & {$[\mathrm{~A} 5 \mathrm{~V}]$} \\
\hline & NGC 234337 & CSI-10-07055 3 & 13.1 & [A8?] \\
\hline & HD 54304 & BD-10 1882 & 9.9 & A \\
\hline & NGC 234327 & CSI-10-07056 4 & 12.4 & {$[\mathrm{~A} 5 / 6 \mathrm{~V}]$} \\
\hline & NGC 234326 & CSI-10-07057 4 & 12.3 & [A7] \\
\hline & NGC 23436 & CSI-10-07057 1 & 10.3 & [B8?] \\
\hline & NGC 234336 & CSI-10-07058 8 & 13.0 & [A7/8?] \\
\hline & NGC 234317 & CSI-10-07057 3 & 11.7 & {$[\mathrm{~A} 2 / 3 \mathrm{~V}]$} \\
\hline & NGC 234310 & CSI-10-07057 2 & 10.5 & [B8/9?] \\
\hline & NGC 234331 & CSI-10-07058 7 & 12.6 & [A6] \\
\hline & BD-10 1883B & - & & \\
\hline & BD-10 1883A & NGC 23435 & 10.1 & [B9] \\
\hline & NGC 234318 & CSI-10-07058 1 & 11.4 & {$[\mathrm{~A} 4 \mathrm{~V}]$} \\
\hline & NGC 234338 & CSI-10-07058 6 & 13.0 & {$[\mathrm{~A} 7 / 8]$} \\
\hline & HD 54360 & BD-10 1884 & 9.4 & A0 \\
\hline & BD-10 1885B & NGC 234311 & 10.7 & B9/A0? \\
\hline & HD 54388 & BD-10 1887 & 8.4 & A3 \\
\hline \multirow[t]{2}{*}{ NGC 2422} & HD 60940 & BD-14 2012 & 8.7 & B7/B8III \\
\hline & HD 60996 & BD-14 2019 & 8.7 & BV \\
\hline NGC 2451 & HD 62974 & CD-37 3855 & 8.3 & $\mathrm{~A} 3 \mathrm{~V}$ \\
\hline NGC 2489 & NGC 248959 & GSC 07119-01134 & 12.9 & B9 \\
\hline \multirow[t]{7}{*}{ NGC 2516} & HD 65691 & NGC 2516 DAC 9 & 9.0 & $\mathrm{~B} 8 / \mathrm{B} 9 \mathrm{~V}$ \\
\hline & CPD-60 942 & NGC 2516 DAC 10 & 10.1 & A1V \\
\hline & CD-60 1929 & NGC 2516 DAC 12 & 8.5 & B9III \\
\hline & CD-60 1932 & NGC 2516 DAC 14 & 9.9 & $\mathrm{~A} 0 \mathrm{~V}$ \\
\hline & HD 65869 & CD-60 1937 & 7.7 & B9V \\
\hline & HD 65896 & NGC 2516 DAC 18 & 9.4 & $\mathrm{~A} 0 \mathrm{~V}$ \\
\hline & HD 65950 & NGC 2516 DAC 27 & 6.9 & B8III \\
\hline
\end{tabular}


S. Bagnulo et al.: A survey of magnetic fields in open cluster A- and B-type stars with FORS1. I., Online Material p 6

Table A.2. continued.

\begin{tabular}{|c|c|c|c|c|}
\hline$\overline{\text { Cluster }}$ & $\overline{\mathrm{ID} 1}$ & $\overline{\mathrm{ID} 2}$ & $\overline{\bar{V}}$ & Spectral Type \\
\hline & V373 Car & NGC 2516 DAC 28 & 9.0 & $\mathrm{~B}$ \\
\hline & HD 65949 & NGC 2516 DAC 26 & 8.4 & B8/B9 \\
\hline & BD-60 969 & NGC 2516 DAC 29 & 8.6 & B9.5IV \\
\hline & BD-60 975 & NGC 2516 DAC 504 & 8.9 & Avar \\
\hline & CPD-60 977 & NGC 2516 DAC 211 & 10.4 & F0V \\
\hline & V410 Car & NGC 2516 DAC 81 & 10.7 & A7V \\
\hline & V392 Car & NGC 2516 DAC 37 & 9.5 & $\mathrm{~A} 2 \mathrm{~V}$ \\
\hline & CD-60 1967 & NGC 2516 DAC 38 & 7.2 & B9.5IV \\
\hline & CD-60 1971 & NGC 2516 DAC 42 & 8.1 & $\mathrm{~B} 8.5 \mathrm{~V}$ \\
\hline & V417 Car & NGC 2516 DAC 40 & 10.4 & {$[\mathrm{~A} 6 \mathrm{~V}]$} \\
\hline & CPD-60 984 & NGC 2516 DAC 41 & 9.7 & $\mathrm{~A} 2 \mathrm{~V}$ \\
\hline & CPD-60 986 & NGC 2516 DAC 901 & 10.1 & {$[\mathrm{~A} 2 \mathrm{~V}]$} \\
\hline & V418 Car & NGC 2516 DAC 43 & 11.0 & {$[\mathrm{~A} 6 / 7 \mathrm{~V}]$} \\
\hline & CD-60 1974 & NGC 2516 DAC 607 & 9.4 & A1V \\
\hline & CD-60 1975 & NGC 2516 SBL 753 & 8.4 & B9V \\
\hline & CD-60 1976 & NGC 2516 DAC 48 & 9.7 & $\mathrm{~A} 0 \mathrm{~V}$ \\
\hline & CD-60 1979 & NGC 2516 DAC 615 & 10.8 & $\mathrm{~A} 3 \mathrm{~V}$ \\
\hline & CD-60 1978 & NGC 2516 DAC 49 & 8.8 & B8.5IV-V \\
\hline & CD-60 1981 & NGC 2516 DAC 608 & 10.7 & $\mathrm{~A} 1 \mathrm{Vm}$ \\
\hline & V420 Car & NGC 2516 DAC 51 & 10.4 & $\mathrm{~A} 3 \mathrm{~V}$ \\
\hline & HD 66137 & NGC 2516 DAC 53 & 7.8 & B9V: \\
\hline & HD 66194 & V374 Car & 5.8 & B2IVnpe \\
\hline & CD-60 1996 & NGC 2516 DAC 806 & 10.8 & $\mathrm{~A} 8 \mathrm{~V}$ \\
\hline & CD-60 1999 & NGC 2516 DAC 60 & 10.0 & $\mathrm{~A} 2 \mathrm{~V}$ \\
\hline \multirow[t]{5}{*}{ NGC 2546} & [N75] 195 & GSC 07133-00458 & 10.5 & B3: \\
\hline & [N75] 196 & - & 10.7 & B3: \\
\hline & NGC 2546272 & GEN \#2.25460272 & 10.7 & B3 \\
\hline & CD-37 4353 & CPD-37 1983 & $?$ & $?$ \\
\hline & HD 69003 & CD-37 4413 & 8.8 & A0 \\
\hline \multirow[t]{2}{*}{ NGC 2925} & HD 298537 & CPD-52 2517 & 11.0 & A \\
\hline & HD 298536 & CPD-52 2522 & - & $\mathrm{AO}$ \\
\hline \multirow{4}{*}{ NGC 3114} & NGC 3114233 & - & - & - \\
\hline & CPD-59 1698 & NGC 311454 & 10.8 & - \\
\hline & CPD-59 1700 & NGC 311456 & 11.1 & - \\
\hline & CPD-59 1703 & NGC 311465 & 11.0 & - \\
\hline \multirow[t]{11}{*}{ NGC 3228} & HD 298051 & CD-51 4686 & 10.2 & $\mathrm{~A} 1 \mathrm{~V}$ \\
\hline & HD 89901 & V343 Vel & 8.4 & B8/B9III \\
\hline & HD 89900 & CD-51 4691 & 8.2 & A0/A1IV/V \\
\hline & HD 89915 & CD-51 4693 & 7.8 & B9.5V \\
\hline & HD 89922 & CD-51 4696 & 9.3 & A4IV/V \\
\hline & HD 298047 & CD-51 4695 & 9.2 & B9V \\
\hline & HD 89938 & CD-51 4698 & 9.3 & A \\
\hline & HD 89937 & CD-51 4699 & 8.9 & B6/B7 \\
\hline & HD 89956 & CD-51 4701 & 8.2 & B9IV/V \\
\hline & CPD-51 3249 & NGC 322815 & 11.3 & {$[\mathrm{~A} 8 \mathrm{~V}]$} \\
\hline & HD 298053 & CD-51 4702 & 10.6 & $\mathrm{~A} 3 \mathrm{~m}$ \\
\hline vdB-Hagen 88 & HD 303107 & HD 92234 & 9.6 & B9 \\
\hline \multirow[t]{2}{*}{ Collinder 228} & HD 305535 & Collinder 22825 & 9.4 & $\mathrm{~B} 2.5 \mathrm{Vn}$ \\
\hline & HD 305543 & DW Car & 10.0 & B5Iab \\
\hline \multirow[t]{3}{*}{ NGC 3532} & HD 96653 & CD-58 3835 & 8.4 & A0III \\
\hline & NGC 3532447 & - & 9.7 & [A5] \\
\hline & HD 96790 & CD-57 3718 & 10.1 & B \\
\hline \multirow[t]{2}{*}{ NGC 5460} & CD-47 8868 & NGC 546036 & 10.7 & $\mathrm{~A} 0$ \\
\hline & HD 123201B & CD-47 8899B & 9.1 & A0 \\
\hline NGC 5662 & HD 127835 & CD-55 5723 & 9.4 & $\mathrm{~B} 8 \mathrm{~V}$ \\
\hline
\end{tabular}


S. Bagnulo et al.: A survey of magnetic fields in open cluster A- and B-type stars with FORS1. I., Online Material p 7

Table A.2. continued.

\begin{tabular}{|c|c|c|c|c|}
\hline Cluster & ID1 & ID2 & $\bar{V}$ & Spectral Type \\
\hline & CPD-56 6334 & NGC 5662104 & 9.9 & B9V \\
\hline & NGC 5662126 & CPD-56 6337 & 10.7 & A1V \\
\hline & HD 127900 & CD-55 5726 & 8.8 & B8II/III \\
\hline & HD 127924 & CD-55 5727 & 9.2 & B8III/IV \\
\hline \multirow{3}{*}{ NGC 6087} & NGC 6087129 & GEN\# + 2.60870129 & 11.5 & - \\
\hline & TYC 8719- 717-1 & GSC 08719-00717 & 10.2 & [A0] \\
\hline & HD 146484 & СРC 205173 & 9.5 & $\mathrm{~A} 0$ \\
\hline \multirow[t]{2}{*}{ NGC 6193} & CD-48 11050 & CPD-48 8680 & 10.3 & $\mathrm{~A} 2$ \\
\hline & CD-48 11060 & CPD-48 8694 & 10.7 & B3V \\
\hline NGC 6281 & HD 323673 & CD-37 11212 & 10.2 & A5 \\
\hline \multirow[t]{6}{*}{ NGC 6383} & NGC 638328 & GEN\# + 2.63830028 & 12.5 & A \\
\hline & NGC 6383700 & GEN\# +2.63830700 & 12.7 & - \\
\hline & NGC 638387 & - & 13.7 & - \\
\hline & HD 317846 & CPD-32 4611 & 9.9 & B5 \\
\hline & NGC 6383102 & GEN\# + 2.63830102 & 14.6 & - \\
\hline & HD 317852 & NGC 638321 & 11.9 & $\mathrm{~A} 0$ \\
\hline \multirow[t]{9}{*}{ NGC 6405} & HD 318108 & CD-32 13080 & 9.7 & B9 \\
\hline & HD 318109 & - & 9.9 & A0 \\
\hline & CD-32 13089 & NGC 640553 & 9.9 & [A4] \\
\hline & CD-32 13093 & NGC 640552 & 10.2 & [A0] \\
\hline & V976 Sco & NGC 640531 & 11.5 & [A?] \\
\hline & HD 318099 & CD-32 13111 & 9.9 & A0 \\
\hline & HD 320765 & CD-34 12156 & 8.8 & $\mathrm{~A} 2$ \\
\hline & HD 162678 & CD-34 12219 & 6.4 & B9V \\
\hline & HD 162724 & V906 Sco & 6.0 & $\mathrm{~B} 9 \mathrm{~V}+$ \\
\hline \multirow[t]{4}{*}{ IC 4725} & BD-19 5044 F & $?$ & 9.7 & B8V \\
\hline & BD-19 5045? & CPD-19 6899 & 9.1 & B5 \\
\hline & BD-19 $5044 \mathrm{M}$ & CPD-19 6901 & 10.2 & B8V \\
\hline & HD 170835 & BD-19 5055 & 8.8 & B5IV \\
\hline
\end{tabular}


Table A.3. $\left\langle B_{z}\right\rangle$ measurements for chemically peculiar stars. Columns 2 and 3 give the date and UT at mid-exposure of the observation, respectively. Columns 4-6 report the $\left\langle B_{z}\right\rangle$ measurement from the Balmer lines, the metal lines, and the full spectrum, as explained in the text. Column 7 reports the flag for the $\left\langle B_{z}\right\rangle$ detection obtained from Balmer, metal lines, and full spectrum. The meaning of "D", "d", and " $n$ " is explained in the text, in Sect. 7. Column 8 reports the signal to noise ratio (SNR) per $\AA$ calculated, for stars observed with grism $600 \mathrm{~B}$, in the wavelength interval 4975-5025 $\AA$ (close to the red wing of $\mathrm{H} \beta$ ). For stars observed with grism $600 \mathrm{R}$, the SNR was calculated in the wavelength interval 6640-6690 $\AA$ (i.e., close to the red wing of $\mathrm{H} \alpha$ ). Column 9 is used to report flags and notes. Unless otherwise marked, the star was observed with grism $600 \mathrm{~B}$.

\begin{tabular}{|c|c|c|c|c|c|c|c|c|}
\hline ID1 & Date & UT & $\begin{array}{c}\left\langle B_{z}\right\rangle(\mathrm{G}) \\
\text { Balmer lines }\end{array}$ & $\begin{array}{c}\left\langle B_{z}\right\rangle(\mathrm{G}) \\
\text { metal lines }\end{array}$ & $\begin{array}{c}\left\langle B_{z}\right\rangle(\mathrm{G}) \\
\text { full spectrum }\end{array}$ & Flag & SNR & Note \\
\hline HD 225264 & $2004-07-31$ & 09:49:40 & $-48 \pm 38$ & $71 \pm 72$ & $-23 \pm 32$ & nnn & 3900 & \\
\hline \multirow[t]{2}{*}{ HD 30598} & 2004-09-01 & $07: 42: 15$ & $124 \pm 120$ & $-55 \pm 143$ & $49 \pm 90$ & nnn & 1600 & \\
\hline & 2005-01-29 & $01: 54: 58$ & $121 \pm 65$ & $89 \pm 94$ & $118 \pm 52$ & nnd & 2500 & \\
\hline HD 35008 & $2005-01-29$ & $00: 22: 02$ & $-340 \pm 72$ & $-273 \pm 293$ & $-339 \pm 69$ & $\mathrm{DnD}$ & 2200 & $1, M$ (new) \\
\hline HD 36046 & 2005-01-30 & 01:21:07 & $-27 \pm 83$ & $-110 \pm 175$ & $-39 \pm 74$ & nnn & 2000 & \\
\hline HD 36540 & 2003-02-08 & 01:40:07 & $301 \pm 67$ & $187 \pm 115$ & $277 \pm 56$ & $\mathrm{dnD}$ & 2700 & $1, M($ new) \\
\hline HD 36549 & $2005-01-30$ & 02:01:45 & $-57 \pm 80$ & $-206 \pm 223$ & $-57 \pm 74$ & $\mathrm{nnn}$ & 1800 & \\
\hline HD 36629 & 2003-02-08 & 02:08:23 & $87 \pm 66$ & $-58 \pm 73$ & $15 \pm 52$ & nnn & 2600 & 2 \\
\hline HD 36918 & 2003-02-09 & 02:10:05 & $-13 \pm 58$ & $31 \pm 150$ & $6 \pm 54$ & nnn & 2700 & \\
\hline HD 36916 & 2003-02-09 & 01:00:16 & $-539 \pm 56$ & $-261 \pm 123$ & $-488 \pm 51$ & DnD & 2700 & 3 , \\
\hline HD 36960 & 2003-02-09 & $01: 42: 20$ & $-90 \pm 63$ & $-132 \pm 64$ & $-115 \pm 45$ & nnn & 2900 & \\
\hline HD 36982 & 2003-02-08 & 01:11:00 & $238 \pm 239$ & $-192 \pm 271$ & $91 \pm 193$ & nnn & 750 & \\
\hline HD 290665 & $2005-01-29$ & 01:20:42 & $-1778 \pm 67$ & $-1588 \pm 64$ & $-1664 \pm 44$ & DDD & 2250 & M (new) \\
\hline HD 37022 & 2003-02-08 & 01:11:00 & $57 \pm 82$ & $115 \pm 82$ & $86 \pm 49$ & nnn & 4150 & \\
\hline HD 37058 & 2003-02-09 & 00:41:09 & $-975 \pm 77$ & $-664 \pm 87$ & $-812 \pm 59$ & DDD & 2100 & $4, \mathrm{M}$ \\
\hline HD 37210 & 2003-02-09 & $01: 21: 46$ & $29 \pm 66$ & $-157 \pm 117$ & $-30 \pm 57$ & nnn & 2250 & 1 \\
\hline HD 37333 & 2005-01-29 & $00: 44: 54$ & $23 \pm 63$ & $-261 \pm 117$ & $-46 \pm 55$ & nnn & 2600 & \\
\hline HD 37470 & 2003-02-08 & 02:49:06 & $-62 \pm 66$ & $-185 \pm 166$ & $-62 \pm 60$ & nnn & 2450 & 1 \\
\hline HD 37633 & 2005-01-30 & $01: 41: 35$ & $295 \pm 83$ & $292 \pm 97$ & $283 \pm 62$ & DdD & 2000 & $M$ (new) \\
\hline V1356 Ori & $2003-02-08$ & $03: 40: 54$ & $-3408 \pm 144$ & $-1290 \pm 180$ & $-2449 \pm 113$ & DDD & 1250 & $M$ (new) \\
\hline HD 45583 & 2003-09-09 & $05: 40: 42$ & $-1518 \pm 71$ & $-1370 \pm 58$ & $-1433 \pm 44$ & DDD & 2500 & $M($ new) \\
\hline NGC 2244334 & 2003-02-09 & $03: 52: 60$ & $-9515 \pm 196$ & $-4815 \pm 136$ & $-6215 \pm 112$ & DDD & 1100 & $5, \mathrm{M}$ (new) \\
\hline HD 49023 & 2005-01-30 & $02: 21: 14$ & $79 \pm 70$ & $-48 \pm 244$ & $100 \pm 68$ & nnn & 2000 & \\
\hline CPD-20 1640 & 2005-01-30 & $03: 35: 37$ & $204 \pm 92$ & $40 \pm 101$ & $157 \pm 63$ & dnn & 1850 & \\
\hline \multirow[t]{2}{*}{ HD 49299} & 2004-09-28 & $08: 26: 26$ & $-483 \pm 42$ & $-508 \pm 37$ & $-659 \pm 83$ & DDD & 3300 & M (new) \\
\hline & 2005-01-30 & $02: 50: 57$ & $-2720 \pm 60$ & $-2311 \pm 77$ & $-2598 \pm 45$ & DDD & 2200 & M \\
\hline \multirow[t]{2}{*}{ HD 51088} & 2004-09-21 & 09:13:05 & $-118 \pm 51$ & $103 \pm 103$ & $-76 \pm 45$ & nnn & 2900 & \\
\hline & 2005-01-30 & $05: 22: 22$ & $-5 \pm 74$ & $384 \pm 135$ & $102 \pm 64$ & ndd & 1900 & \\
\hline HD 52965 & 2005-01-30 & $05: 03: 48$ & $-69 \pm 92$ & $-105 \pm 217$ & $-64 \pm 84$ & nnn & 1800 & \\
\hline HD 56343 & 2004-09-21 & 08:32:57 & $-3609 \pm 69$ & $-3144 \pm 90$ & $-3415 \pm 54$ & DDD & 1950 & M (new) \\
\hline BD-14 2015 & 2005-01-29 & $05: 47: 41$ & $-31 \pm 72$ & $-290 \pm 258$ & $-32 \pm 69$ & nnn & 2400 & \\
\hline \multirow[t]{2}{*}{ BD-14 2028} & 2004-02-28 & $04: 16: 33$ & $103 \pm 112$ & $-271 \pm 218$ & $32 \pm 93$ & nnn & 1550 & \\
\hline & 2004-03-08 & $04: 45: 24$ & $119 \pm 130$ & $-394 \pm 240$ & $-7 \pm 104$ & nnn & 1400 & \\
\hline \multirow[t]{2}{*}{ HD 61045} & $2003-02-08$ & $04: 28: 15$ & $412 \pm 63$ & $476 \pm 108$ & $506 \pm 111$ & Ddd & 2500 & M (new) \\
\hline & 2005-01-29 & $06: 11: 00$ & $375 \pm 70$ & $186 \pm 137$ & $340 \pm 62$ & $\mathrm{DnD}$ & 2300 & M \\
\hline \multirow[t]{2}{*}{ BD-14 2040} & $2003-02-08$ & $04: 28: 15$ & $169 \pm 273$ & $-149 \pm 244$ & $16 \pm 166$ & nnn & 750 & \\
\hline & 2005-01-29 & 05:04:50 & $56 \pm 72$ & $-104 \pm 101$ & $-37 \pm 55$ & nnn & 2300 & \\
\hline HD 62376 & 2005-01-29 & $02: 41: 28$ & $101 \pm 62$ & $-4 \pm 127$ & $73 \pm 55$ & nnn & 2450 & \\
\hline CD-37 3845 & 2003-02-08 & $04: 52: 12$ & $98 \pm 113$ & $-430 \pm 201$ & $10 \pm 97$ & ndn & 1450 & \\
\hline HD 62992 & 2003-02-08 & $04: 52: 12$ & $-190 \pm 65$ & $-157 \pm 54$ & $-164 \pm 39$ & ddd & 2450 & $M($ new) \\
\hline HD 63079 & 2005-01-29 & $02: 21: 48$ & $8 \pm 64$ & $150 \pm 152$ & $47 \pm 58$ & nnn & 2300 & \\
\hline \multirow[t]{2}{*}{ HD 63401} & 2003-02-08 & $00: 36: 57$ & $-532 \pm 61$ & $-551 \pm 119$ & $-527 \pm 53$ & DDD & 2900 & $6, M($ new) \\
\hline & $2005-01-29$ & $02: 59: 47$ & $357 \pm 96$ & $472 \pm 195$ & $348 \pm 85$ & Dnd & 2450 & M \\
\hline NGC 248958 & 2005-01-30 & $06: 10: 58$ & $-325 \pm 205$ & $-826 \pm 313$ & $-449 \pm 170$ & nnn & 1200 & \\
\hline NGC 248940 & 2005-01-30 & $06: 10: 58$ & $-489 \pm 220$ & $36 \pm 251$ & $-387 \pm 158$ & nnn & 850 & \\
\hline \multirow[t]{2}{*}{ HD 65712} & 2003-02-09 & $07: 20: 21$ & $-1025 \pm 84$ & $-1181 \pm 73$ & $-1111 \pm 54$ & DDD & 1800 & $M($ new) \\
\hline & 2005-01-29 & $04: 22: 56$ & $-668 \pm 55$ & $-357 \pm 74$ & $-558 \pm 43$ & DDD & 2450 & $\mathrm{M}$ \\
\hline \multirow[t]{2}{*}{ CPD-60944A } & 2002-02-05 & 06:03:02 & $-41 \pm 104$ & $122 \pm 87$ & $48 \pm 67$ & nnn & 1950 & $7, \mathrm{R}$ \\
\hline & 2005-01-29 & 03:25:09 & $-54 \pm 58$ & $-74 \pm 113$ & $-50 \pm 51$ & nnn & 2500 & 7 \\
\hline \multirow[t]{2}{*}{ CPD-60944B } & $2002-02-05$ & $06: 55: 01$ & $274 \pm 111$ & $349 \pm 67$ & $349 \pm 66$ & $\mathrm{nDD}$ & 1450 & $7, \mathrm{R}, \mathrm{m}$ (new?) \\
\hline & 2005-01-29 & $03: 52: 30$ & $143 \pm 63$ & $93 \pm 89$ & $158 \pm 50$ & nnd & 2650 & 7 \\
\hline CPD-60 978 & 2002-02-04 & 06:08:09 & $48 \pm 103$ & $-35 \pm 71$ & $2 \pm 58$ & nnn & 1750 & $\mathrm{R}$ \\
\hline
\end{tabular}


S. Bagnulo et al.: A survey of magnetic fields in open cluster A- and B-type stars with FORS1. I., Online Material p 9

Table A.3. continued.

\begin{tabular}{|c|c|c|c|c|c|c|c|c|}
\hline ID1 & Date & UT & $\begin{array}{c}\left\langle B_{z}\right\rangle(\mathrm{G}) \\
\text { Balmer lines }\end{array}$ & $\begin{array}{c}\left\langle B_{z}\right\rangle(\mathrm{G}) \\
\text { metal lines }\end{array}$ & $\begin{array}{c}\left\langle B_{z}\right\rangle(\mathrm{G}) \\
\text { full spectrum }\end{array}$ & Flag & SNR & Note \\
\hline & $2002-02-05$ & $04: 19: 29$ & $58 \pm 83$ & $39 \pm 71$ & $46 \pm 54$ & $\mathrm{nnn}$ & 1850 & $\mathrm{R}$ \\
\hline & 2004-03-08 & $05: 25: 21$ & $-91 \pm 103$ & $-229 \pm 189$ & $-83 \pm 88$ & nnn & 1650 & \\
\hline \multirow[t]{2}{*}{ HD 65987} & 2002-02-04 & $07: 54: 30$ & $629 \pm 162$ & $573 \pm 142$ & $605 \pm 106$ & DDD & 1400 & $\mathrm{R}, \mathrm{M}$ (new) \\
\hline & 2004-02-28 & 05:13:09 & $-443 \pm 70$ & $-507 \pm 105$ & $-460 \pm 58$ & DDD & 2150 & M \\
\hline \multirow[t]{2}{*}{ HD 66295} & 2002-02-05 & 05:08:50 & $-547 \pm 84$ & $-515 \pm 70$ & $-534 \pm 54$ & DDD & 2000 & $\mathrm{R}, \mathrm{M}$ (new) \\
\hline & 2003-02-09 & 06:30:09 & $499 \pm 73$ & $421 \pm 46$ & $438 \pm 38$ & DDD & 2350 & $\mathrm{R}, \mathrm{M}$ \\
\hline \multirow[t]{2}{*}{ HD 66318} & 2002-02-05 & 05:08:50 & $4410 \pm 105$ & $5129 \pm 40$ & $5044 \pm 37$ & DDD & 1600 & $8, \mathrm{R}, \mathrm{M}$ (new) \\
\hline & 2003-02-09 & 06:30:09 & $4266 \pm 85$ & $5322 \pm 31$ & $5196 \pm 29$ & DDD & 1850 & $\mathrm{R}, \mathrm{M}$ \\
\hline \multirow[t]{2}{*}{ NGC 2546258} & 2004-09-27 & 07:57:06 & $-196 \pm 102$ & $-454 \pm 145$ & $-257 \pm 70$ & ndD & 1657 & \\
\hline & 2005-01-30 & $07: 12: 58$ & $-83 \pm 98$ & $16 \pm 78$ & $-21 \pm 55$ & nnn & 1850 & \\
\hline NGC 2546201 & 2005-01-29 & $06: 52: 31$ & $307 \pm 62$ & $355 \pm 57$ & $594 \pm 158$ & DDD & 2650 & M (new) \\
\hline CPD-37 1989 & 2005-01-29 & $06: 52: 31$ & $-94 \pm 91$ & $22 \pm 164$ & $-19 \pm 72$ & nnn & 2050 & \\
\hline HD 69004 & 2004-09-27 & 08:53:02 & $-100 \pm 54$ & $110 \pm 116$ & $-58 \pm 48$ & nnn & 2900 & \\
\hline \multirow[t]{2}{*}{ HD 69067} & 2004-09-29 & $08: 51: 17$ & $518 \pm 64$ & $554 \pm 96$ & $499 \pm 52$ & DdD & 2500 & M (new) \\
\hline & 2004-04-20 & $23: 29: 28$ & $609 \pm 59$ & $377 \pm 78$ & $537 \pm 46$ & DDD & 2300 & M \\
\hline \multirow[t]{2}{*}{ HD 74169} & $2003-02-08$ & $05: 13: 58$ & $-151 \pm 57$ & $-191 \pm 56$ & $-175 \pm 39$ & $\mathrm{ndD}$ & 2250 & m (new?) \\
\hline & 2004-03-02 & $04: 38: 32$ & $-65 \pm 56$ & $-37 \pm 57$ & $-51 \pm 39$ & nnn & 2500 & \\
\hline HD 74535 & $2003-02-08$ & $05: 33: 45$ & $-83 \pm 68$ & $-126 \pm 137$ & $-96 \pm 60$ & nnn & 2250 & \\
\hline HD 75239 & $2005-01-29$ & 08:13:02 & $100 \pm 79$ & $-94 \pm 172$ & $89 \pm 71$ & nnn & 2100 & \\
\hline HD 83002 & 2005-01-30 & $07: 45: 58$ & $5 \pm 80$ & $-102 \pm 161$ & $-18 \pm 70$ & nnn & 2000 & \\
\hline HD 87241 & 2004-05-09 & 01:55:04 & $24 \pm 52$ & $-115 \pm 157$ & $26 \pm 49$ & nnn & 2800 & \\
\hline HD 87240 & 2003-02-08 & $07: 15: 26$ & $-257 \pm 58$ & $-239 \pm 103$ & $-259 \pm 50$ & $\mathrm{DnD}$ & 2500 & M (new) \\
\hline HD 87266 & 2003-02-08 & $06: 21: 28$ & $-45 \pm 38$ & $44 \pm 54$ & $0 \pm 32$ & nnn & 4100 & \\
\hline HD 304841 & 2003-02-08 & $06: 21: 28$ & $-279 \pm 78$ & $-518 \pm 163$ & $-335 \pm 69$ & DDD & 1900 & $M($ new) \\
\hline HD 304842 & $2003-02-08$ & $06: 21: 28$ & $86 \pm 137$ & $-115 \pm 171$ & $-18 \pm 104$ & nnn & 2200 & \\
\hline HD 87405 & 2003-02-08 & $06: 21: 28$ & $-82 \pm 45$ & $2 \pm 65$ & $-61 \pm 36$ & nnn & 3850 & \\
\hline HD 89856 & 2002-02-04 & 09:19:41 & $-361 \pm 112$ & $-90 \pm 91$ & $-197 \pm 70$ & Dnd & 1650 & $\mathrm{R}, \mathrm{M}$ (new) \\
\hline HD 92190 & $2005-01-30$ & $09: 16: 30$ & $21 \pm 79$ & $-42 \pm 149$ & $10 \pm 70$ & nnn & 2250 & \\
\hline HD 92385 & $2005-01-29$ & $09: 28: 11$ & $-580 \pm 60$ & $-266 \pm 142$ & $-519 \pm 55$ & DnD & 2550 & M (new) \\
\hline Cr 22830 & $2004-05-28$ & 00:02:29 & $692 \pm 143$ & $405 \pm 212$ & $558 \pm 113$ & DnD & 1550 & M (new) \\
\hline HD 305451 & 2004-05-28 & 00:02:29 & $-181 \pm 89$ & $-96 \pm 258$ & $-149 \pm 87$ & nnn & 1950 & \\
\hline HD 96040 & $2005-01-29$ & 08:48:39 & $-307 \pm 54$ & $-145 \pm 93$ & $-257 \pm 47$ & DnD & 2350 & $M($ new) \\
\hline HD 96729 & 2004-07-12 & $23: 52: 56$ & $1125 \pm 71$ & $601 \pm 100$ & $949 \pm 57$ & DDD & 2100 & $M($ new) \\
\hline HD 303821 & 2005-01-30 & $08: 35: 12$ & $-74 \pm 88$ & $-113 \pm 84$ & $-100 \pm 60$ & nnn & 1750 & \\
\hline HD 122983 & 2004-08-03 & 01:01:32 & $156 \pm 54$ & $210 \pm 118$ & $170 \pm 48$ & $\mathrm{dnD}$ & 3050 & \\
\hline HD 123183 & 2004-07-13 & $00: 32: 44$ & $-440 \pm 146$ & $-181 \pm 473$ & $-409 \pm 176$ & Dnn & 1300 & m (new?) \\
\hline HD 123225 & $2004-07-13$ & $00: 32: 44$ & $97 \pm 83$ & $-176 \pm 200$ & $-22 \pm 72$ & nnn & 2400 & \\
\hline CPD-56 6330 & 2003-02-08 & $08: 22: 37$ & $-108 \pm 125$ & $-404 \pm 165$ & $-191 \pm 99$ & nnn & 1350 & \\
\hline HD 127866 & $2003-02-08$ & $07: 52: 56$ & $-238 \pm 148$ & $-124 \pm 189$ & $-89 \pm 134$ & nnn & 1250 & \\
\hline CPD-57 7817 & 2004-07-13 & 02:04:24 & $-673 \pm 69$ & $-374 \pm 130$ & $-612 \pm 60$ & DnD & 2550 & $M$ (new) \\
\hline HD 146555 & 2003-02-09 & 08:54:01 & $461 \pm 129$ & $232 \pm 165$ & $268 \pm 97$ & dnn & 1350 & m (new?) \\
\hline HD 149257 & 2004-07-13 & $02: 46: 41$ & $247 \pm 93$ & $43 \pm 110$ & $160 \pm 69$ & nnn & 2350 & \\
\hline HD 149277 & 2004-07-13 & $02: 46: 41$ & $2435 \pm 149$ & $1549 \pm 186$ & $2239 \pm 104$ & DDD & 2550 & $M($ new) \\
\hline \multirow[t]{2}{*}{ CD-48 11051} & 2004-07-13 & $03: 28: 19$ & $-2604 \pm 231$ & $-1293 \pm 283$ & $-2013 \pm 117$ & DDD & 1400 & $M$ (new) \\
\hline & 2004-07-24 & $02: 51: 35$ & $-2173 \pm 105$ & $-801 \pm 162$ & $-1772 \pm 74$ & DDD & 2050 & M \\
\hline \multirow[t]{2}{*}{ CD-48 11059} & $2004-07-13$ & $03: 28: 19$ & $217 \pm 200$ & $-15 \pm 433$ & $172 \pm 173$ & nnn & 1000 & \\
\hline & 2004-07-24 & $02: 51: 35$ & $193 \pm 138$ & $-145 \pm 183$ & $90 \pm 107$ & nnn & 1400 & \\
\hline HD 322676 & 2004-08-02 & $05: 36: 38$ & $-277 \pm 117$ & $246 \pm 213$ & $-191 \pm 102$ & nnn & 2150 & \\
\hline HD 153948 & 2004-08-02 & $05: 36: 38$ & $199 \pm 58$ & $176 \pm 141$ & $195 \pm 53$ & $\mathrm{DnD}$ & 3100 & $M($ new) \\
\hline NGC 638326 & 2004-08-03 & $04: 21: 50$ & $-275 \pm 217$ & $303 \pm 251$ & $48 \pm 152$ & nnn & 1200 & \\
\hline HD 317857 & 2004-07-24 & $03: 52: 22$ & $-1677 \pm 75$ & $-1393 \pm 81$ & $-1558 \pm 54$ & DDD & 2150 & $M($ new) \\
\hline HD 318107 & $2004-07-25$ & $23: 40: 24$ & $6519 \pm 55$ & $3784 \pm 59$ & $5216 \pm 39$ & DDD & 2600 & $9, \mathrm{M}$ \\
\hline HD 318100 & 2004-08-17 & $01: 20: 22$ & $390 \pm 71$ & $93 \pm 151$ & $345 \pm 64$ & DnD & 2100 & $M($ new) \\
\hline CD-32 13119 & 2004-08-03 & $02: 21: 41$ & $-29 \pm 65$ & $-39 \pm 37$ & $-37 \pm 30$ & nnn & 2450 & \\
\hline HD 318095 & 2004-08-03 & $02: 21: 41$ & $101 \pm 64$ & $-168 \pm 138$ & $42 \pm 54$ & nnn & 2658 & \\
\hline \multirow[t]{2}{*}{ HD 162305} & 2004-08-03 & $06: 18: 16$ & $1 \pm 40$ & $122 \pm 95$ & $24 \pm 37$ & nnn & 3950 & \\
\hline & $2004-09-26$ & $02: 18: 57$ & $112 \pm 72$ & $230 \pm 150$ & $120 \pm 65$ & nnn & 2150 & \\
\hline HD 320764 & 2004-08-23 & $01: 44: 37$ & $-89 \pm 78$ & $-43 \pm 154$ & $-69 \pm 66$ & nnn & 2400 & \\
\hline HD 162725 & 2004-08-02 & $06: 36: 03$ & $-67 \pm 35$ & $37 \pm 115$ & $-60 \pm 33$ & nnn & 4450 & \\
\hline
\end{tabular}


Table A.3. continued.

\begin{tabular}{lccccclll}
\hline \hline & & & $\left\langle B_{z}\right\rangle(\mathrm{G})$ & $\left\langle B_{z}\right\rangle(\mathrm{G})$ & $\left\langle B_{z}\right\rangle(\mathrm{G})$ & & \\
\multicolumn{1}{c}{ ID1 } & Date & $\mathrm{UT}$ & Balmer lines & metal lines & full spectrum & Flag & SNR & Note \\
\hline HD 169959 & $2004-07-06$ & $03: 48: 34$ & $-541 \pm 58$ & $-302 \pm 128$ & $-486 \pm 52$ & DnD & 2550 & M(new) \\
HD 170054 & $2004-08-27$ & $02: 43: 53$ & $16 \pm 72$ & $361 \pm 153$ & $64 \pm 68$ & nnn & 2300 & \\
& $2004-09-23$ & $01: 21: 38$ & $254 \pm 100$ & $-207 \pm 136$ & $84 \pm 77$ & nnn & 2200 & \\
BD-19 5044L & $2004-08-23$ & $02: 34: 33$ & $-223 \pm 123$ & $-347 \pm 341$ & $-235 \pm 111$ & nnn & 1550 & \\
BD-19 5046 & $2004-08-23$ & $02: 34: 33$ & $68 \pm 100$ & $64 \pm 64$ & $60 \pm 92$ & nnn & 2650 & \\
HD 170836 & $2004-08-23$ & $03: 26: 34$ & $-636 \pm 135$ & $-193 \pm 220$ & $-487 \pm 114$ & DnD & 1350 & M (new) \\
& $2004-08-28$ & $04: 37: 57$ & $-633 \pm 100$ & $-449 \pm 193$ & $-583 \pm 88$ & DdD & 1950 & M \\
HD 170860A & $2004-09-26$ & $02: 54: 12$ & $502 \pm 95$ & $436 \pm 140$ & $483 \pm 77$ & dDD & 2100 & M \\
HD 190290 & $2004-08-04$ & $06: 40: 14$ & $-70 \pm 79$ & $62 \pm 152$ & $-41 \pm 69$ & nnn & 2050 & \\
\hline
\end{tabular}

R: observed with grism $600 \mathrm{R}$.

M: definite detection according to our judgement.

(new): Star previously not known as magnetic.

$\mathrm{m}$ : marginal detection according to our judgement.

1: four null detections by Borra (1994; pr. comm. cited in Bychkov et al. 2003).

2: Sargent et al. (1967) reported $\left\langle B_{z}\right\rangle=1300 \pm 400 \mathrm{G}$. Several additional measurements by Conti (1970) suggest that $\left\langle B_{z}\right\rangle$ varies approximately from -700 to $+1400 \mathrm{G}$.

3: Borra et al. (1983) measured $\left\langle B_{z}\right\rangle=628 \pm 178 \mathrm{G}$.

4: Field detected by Sargent et al. (1967), Conti (1970), Borra et al. (1983), Mathys \& Hubrig (1997).

5: field detection reported by Bagnulo et al. (2004).

6: null detection by Bohelender et al. (1993).

7: SIMBAD coordinates are inverted (A with B and B with A). For CpD-60 944A RA and Dec are 07:56:45.3 -60:48:55; for CpD-60 944B RA and Dec are 07:56:46.3 -60:48:59.2.

8: field detection reported by Bagnulo et al. (2003).

9: Mathys \& Hubrig (1997) detected $\left\langle B_{z}\right\rangle=1985 \pm 230 \mathrm{G}$.

10: Hubrig et al. (2004) detected $\left\langle B_{z}\right\rangle=3220 \pm 73 \mathrm{G}$ and $\left\langle B_{z}\right\rangle=3250 \pm 111 \mathrm{G}$. 
S. Bagnulo et al.: A survey of magnetic fields in open cluster A- and B-type stars with FORS1. I., Online Material p 11

Table A.4. $\left\langle B_{z}\right\rangle$ measurements for stars not known to be chemically peculiar stars. Unless otherwise stated in the notes, the observations have been obtained with grism $600 \mathrm{~B}$.

\begin{tabular}{|c|c|c|c|c|c|c|c|c|}
\hline ID1 & Date & UT & $\begin{array}{c}\left\langle B_{z}\right\rangle(\mathrm{G}) \\
\text { Balmer lines }\end{array}$ & $\begin{array}{c}\left\langle B_{z}\right\rangle(\mathrm{G}) \\
\text { metal lines }\end{array}$ & $\begin{array}{c}\left\langle B_{z}\right\rangle(\mathrm{G}) \\
\text { full spectrum }\end{array}$ & Flag & SNR & Note \\
\hline HD 36559 & $2003-02-08$ & 01:40:07 & $-3 \pm 81$ & $-76 \pm 146$ & $-25 \pm 68$ & nnn & 1900 & \\
\hline HD 36671 & 2003-02-08 & 02:08:23 & $69 \pm 136$ & $49 \pm 70$ & $50 \pm 62$ & nnn & 1500 & \\
\hline HD 37041 & 2003-02-08 & 01:11:00 & $125 \pm 92$ & $26 \pm 70$ & $47 \pm 58$ & nnn & 3950 & \\
\hline HD 37428 & 2003-02-08 & 02:49:06 & $-175 \pm 88$ & $-45 \pm 158$ & $-159 \pm 88$ & nnn & 2100 & \\
\hline HD 252214 & 2003-02-08 & $03: 40: 54$ & $-49 \pm 55$ & $-11 \pm 68$ & $-26 \pm 46$ & nnn & 3350 & \\
\hline HD 41909 & 2003-02-08 & $03: 40: 54$ & $5 \pm 49$ & $119 \pm 23$ & $104 \pm 20$ & $\mathrm{nDD}$ & 2900 & \\
\hline HD 252248 & 2003-02-08 & $03: 40: 54$ & $-144 \pm 108$ & $-160 \pm 104$ & $-189 \pm 77$ & nnn & 2800 & \\
\hline HD 252266 & 2003-02-08 & $03: 40: 54$ & $111 \pm 89$ & $-94 \pm 78$ & $-18 \pm 60$ & nnn & 2500 & \\
\hline NGC 2244336 & 2003-02-09 & $03: 52: 60$ & $399 \pm 182$ & $-64 \pm 84$ & $-19 \pm 74$ & nnn & 1250 & \\
\hline CPD-20 1637 & 2004-09-28 & $08: 26: 26$ & $76 \pm 67$ & $-26 \pm 194$ & $88 \pm 62$ & nnn & 2700 & \\
\hline \multirow{2}{*}{ BD-20 1571} & 2004-09-28 & $08: 26: 26$ & $-104 \pm 81$ & $58 \pm 129$ & $-37 \pm 65$ & nnn & 2400 & \\
\hline & $2005-01-30$ & $02: 50: 57$ & $265 \pm 108$ & $55 \pm 156$ & $200 \pm 81$ & dnd & 1650 & \\
\hline NGC 2287 AR 157 & 2005-01-30 & $03: 35: 37$ & $-14 \pm 138$ & $-153 \pm 216$ & $2 \pm 106$ & nnn & 1250 & \\
\hline CPD-20 1645 & $2005-01-30$ & $03: 35: 37$ & $-55 \pm 88$ & $130 \pm 171$ & $-15 \pm 72$ & nnn & 1950 & \\
\hline HD 52980 & $2005-01-30$ & 05:03:48 & $-131 \pm 58$ & $-48 \pm 173$ & $-111 \pm 54$ & nnn & 2450 & \\
\hline BD-08 1708 & $2005-01-30$ & $05: 03: 48$ & $-1 \pm 146$ & $-65 \pm 335$ & $-48 \pm 131$ & nnn & 1200 & \\
\hline CSI-10-07049 & 2002-02-05 & $01: 22: 04$ & $-234 \pm 157$ & $161 \pm 134$ & $-19 \pm 76$ & nnn & 1350 & $\mathrm{R}$ \\
\hline BD-10 1875 & 2002-02-05 & $01: 22: 04$ & $-92 \pm 202$ & $-8 \pm 188$ & $-31 \pm 138$ & nnn & 1550 & $\mathrm{R}$ \\
\hline NGC 234322 & $2002-02-05$ & 01:22:04 & $243 \pm 218$ & $88 \pm 204$ & $242 \pm 150$ & nnn & 1150 & $\mathrm{R}$ \\
\hline NGC 234340 & $2002-02-05$ & 03:00:13 & $215 \pm 238$ & $45 \pm 153$ & $68 \pm 127$ & nnn & 750 & $\mathrm{R}$ \\
\hline CSI-10-07053 5 & 2002-02-05 & $01: 22: 04$ & $320 \pm 263$ & $116 \pm 253$ & $251 \pm 186$ & nnn & 890 & $\mathrm{R}$ \\
\hline BD-10 1878 & $2002-02-05$ & 03:00:13 & $111 \pm 115$ & $-144 \pm 145$ & $12 \pm 89$ & nnn & 1450 & $\mathrm{R}$ \\
\hline NGC 234325 & $2002-02-05$ & 03:00:13 & $280 \pm 246$ & $124 \pm 224$ & $291 \pm 192$ & nnn & 1000 & $\mathrm{R}$ \\
\hline NGC 234316 & $2002-02-05$ & 03:00:13 & $-225 \pm 183$ & $-162 \pm 186$ & $-227 \pm 127$ & nnn & 1350 & $\mathrm{R}$ \\
\hline BD-10 1879a & 2002-02-04 & 03:50:42 & $-178 \pm 228$ & $12 \pm 171$ & $-20 \pm 132$ & nnn & 1350 & $\mathrm{R}$ \\
\hline NGC 234334 & 2002-02-04 & $01: 35: 56$ & $-374 \pm 272$ & $169 \pm 214$ & $-40 \pm 167$ & nnn & 800 & $\mathrm{R}$ \\
\hline NGC 234343 & 2002-02-04 & 05:01:44 & $64 \pm 334$ & $34 \pm 243$ & $10 \pm 181$ & nnn & 700 & $\mathrm{R}$ \\
\hline NGC 234328 & 2002-02-05 & 03:00:13 & $164 \pm 234$ & $24 \pm 181$ & $82 \pm 145$ & nnn & 1050 & $\mathrm{R}$ \\
\hline NGC 234337 & 2002-02-04 & 05:01:44 & $-51 \pm 274$ & $21 \pm 176$ & $-59 \pm 146$ & $\mathrm{nnn}$ & 850 & $\mathrm{R}$ \\
\hline HD 54304 & 2002-02-04 & $02: 57: 20$ & $197 \pm 106$ & $-47 \pm 99$ & $54 \pm 70$ & nnn & 2000 & $\mathrm{R}$ \\
\hline NGC 234327 & 2002-02-04 & 05:01:44 & $40 \pm 262$ & $-103 \pm 212$ & $-32 \pm 164$ & nnn & 1050 & $\mathrm{R}$ \\
\hline NGC 234326 & 2002-02-04 & 05:01:44 & $75 \pm 200$ & $-11 \pm 203$ & $32 \pm 140$ & nnn & 1200 & $\mathrm{R}$ \\
\hline NGC 23436 & 2002-02-04 & $02: 57: 20$ & $-132 \pm 107$ & $113 \pm 121$ & $-32 \pm 80$ & nnn & 1900 & $\mathrm{R}$ \\
\hline NGC 234336 & 2002-02-04 & $01: 35: 56$ & $-226 \pm 260$ & $388 \pm 305$ & $26 \pm 193$ & nnn & 1000 & $\mathrm{R}$ \\
\hline NGC 234317 & 2002-02-04 & $01: 35: 56$ & $50 \pm 167$ & $-11 \pm 212$ & $-13 \pm 118$ & nnn & 1250 & $\mathrm{R}$ \\
\hline NGC 234310 & 2002-02-04 & $02: 57: 20$ & $67 \pm 113$ & $152 \pm 132$ & $122 \pm 85$ & nnn & 1550 & $\mathrm{R}$ \\
\hline NGC 234331 & 2002-02-04 & 05:01:44 & $-71 \pm 280$ & $-530 \pm 284$ & $-296 \pm 192$ & nnn & 750 & $\mathrm{R}$ \\
\hline BD-10 1883B & 2002-02-04 & $02: 57: 20$ & $17 \pm 215$ & $70 \pm 185$ & $55 \pm 139$ & nnn & 1250 & $\mathrm{R}$ \\
\hline BD-10 1883A & 2002-02-04 & $01: 35: 56$ & $-45 \pm 173$ & $-134 \pm 150$ & $-115 \pm 112$ & nnn & 1750 & $\mathrm{R}$ \\
\hline NGC 234318 & 2002-02-04 & $01: 35: 56$ & $76 \pm 176$ & $448 \pm 214$ & $166 \pm 131$ & nnn & 1250 & $\mathrm{R}$ \\
\hline NGC 234338 & 2002-02-04 & $02: 57: 20$ & $187 \pm 352$ & $-123 \pm 243$ & $-23 \pm 198$ & nnn & 700 & $\mathrm{R}$ \\
\hline HD 54360 & 2002-02-04 & $01: 35: 56$ & $-74 \pm 166$ & $-47 \pm 102$ & $-50 \pm 85$ & nnn & 2150 & $\mathrm{R}$ \\
\hline BD-10 1885B & 2002-02-04 & 05:01:44 & $340 \pm 136$ & $-102 \pm 115$ & $89 \pm 88$ & nnn & 1750 & $\mathrm{R}$ \\
\hline HD 54388 & 2002-02-04 & $03: 50: 42$ & $5 \pm 64$ & $-134 \pm 54$ & $-66 \pm 41$ & nnn & 2450 & \\
\hline HD 60940 & 2005-01-29 & $05: 47: 41$ & $6 \pm 50$ & $97 \pm 165$ & $10 \pm 47$ & nnn & 3250 & \\
\hline HD 60996 & 2005-01-29 & $05: 47: 41$ & $61 \pm 58$ & $93 \pm 157$ & $76 \pm 57$ & nnn & 3250 & \\
\hline HD 62974 & 2003-02-08 & $04: 52: 12$ & $-26 \pm 105$ & $-3 \pm 205$ & $-50 \pm 87$ & nnn & 1850 & \\
\hline NGC 248959 & 2005-01-30 & $06: 10: 58$ & $360 \pm 169$ & $1256 \pm 372$ & $517 \pm 181$ & ndn & 1300 & \\
\hline HD 65691 & 2002-02-05 & 06:03:02 & $-158 \pm 215$ & $-344 \pm 172$ & $-262 \pm 134$ & nnn & 1500 & $\mathrm{R}$ \\
\hline CPD-60 942 & 2002-02-05 & 06:03:02 & $616 \pm 281$ & $-208 \pm 216$ & $106 \pm 169$ & nnn & 950 & $\mathrm{R}$ \\
\hline CD-60 1929 & 2002-02-05 & 06:03:02 & $-52 \pm 109$ & $244 \pm 108$ & $110 \pm 76$ & nnn & 2100 & $\mathrm{R}$ \\
\hline \multirow[t]{2}{*}{ CD-60 1932} & 2002-02-05 & $06: 55: 01$ & $-110 \pm 338$ & $-144 \pm 231$ & $-99 \pm 188$ & nnn & 1100 & $\mathrm{R}$ \\
\hline & 2002-02-05 & $06: 03: 02$ & $-28 \pm 305$ & $7 \pm 225$ & $-35 \pm 178$ & nnn & 1200 & $\mathrm{R}$ \\
\hline HD 65869 & $2002-02-05$ & $06: 55: 01$ & $233 \pm 118$ & $-203 \pm 107$ & $-5 \pm 79$ & nnn & 2750 & $\mathrm{R}$ \\
\hline HD 65896 & 2002-02-04 & $07: 54: 30$ & $109 \pm 111$ & $144 \pm 98$ & $127 \pm 73$ & nnn & 1900 & $\mathrm{R}$ \\
\hline HD 65950 & 2002-02-05 & $07: 45: 43$ & $-32 \pm 50$ & $-123 \pm 62$ & $-114 \pm 50$ & nnn & 2800 & $\mathrm{R}$ \\
\hline V373 Car & 2004-03-08 & $05: 25: 21$ & $-187 \pm 75$ & $-34 \pm 61$ & $-104 \pm 48$ & nnn & 1350 & \\
\hline
\end{tabular}


S. Bagnulo et al.: A survey of magnetic fields in open cluster A- and B-type stars with FORS1. I., Online Material p 12

Table A.4. continued.

\begin{tabular}{|c|c|c|c|c|c|c|c|c|}
\hline ID1 & Date & UT & $\begin{array}{c}\left\langle B_{z}\right\rangle(\mathrm{G}) \\
\text { Balmer lines }\end{array}$ & $\begin{array}{c}\left\langle B_{z}\right\rangle(\mathrm{G}) \\
\text { metal lines }\end{array}$ & $\begin{array}{c}\left\langle B_{z}\right\rangle(\mathrm{G}) \\
\text { full spectrum }\end{array}$ & Flag & SNR & Note \\
\hline & $2002-02-05$ & $04: 19: 29$ & $-87 \pm 23$ & $-25 \pm 36$ & $-66 \pm 19$ & $\mathrm{DnD}$ & 1600 & $\mathrm{R}$ \\
\hline HD 65949 & 2002-02-04 & $07: 54: 30$ & $-19 \pm 89$ & $158 \pm 83$ & $74 \pm 61$ & nnn & 2250 & $\mathrm{R}$ \\
\hline BD-60 969 & 2002-02-04 & $07: 54: 30$ & $43 \pm 136$ & $-138 \pm 107$ & $-69 \pm 84$ & nnd & 2350 & $\mathrm{R}$ \\
\hline \multirow{2}{*}{ BD-60 975} & 2002-02-05 & $04: 19: 29$ & $145 \pm 111$ & $100 \pm 111$ & $127 \pm 78$ & nnd & 2250 & $\mathrm{R}$ \\
\hline & 2002-02-04 & 06:08:09 & $65 \pm 123$ & $229 \pm 140$ & $132 \pm 89$ & nnn & 2250 & $\mathrm{R}$ \\
\hline CPD-60 977 & 2002-02-04 & $07: 01: 42$ & $35 \pm 109$ & $75 \pm 94$ & $57 \pm 70$ & nnn & 1550 & $\mathrm{R}$ \\
\hline V410 Car & 2002-02-04 & 07:01:42 & $77 \pm 225$ & $-200 \pm 188$ & $-98 \pm 140$ & $\mathrm{nnn}$ & 1450 & $\mathrm{R}$ \\
\hline \multirow[t]{3}{*}{ V392 Car } & 2004-03-08 & $05: 25: 21$ & $221 \pm 129$ & $-75 \pm 144$ & $111 \pm 91$ & nnn & 1250 & \\
\hline & 2002-02-05 & 04:19:29 & $-4 \pm 97$ & $85 \pm 73$ & $34 \pm 57$ & nnn & 2000 & $\mathrm{R}$ \\
\hline & 2002-02-05 & $07: 45: 43$ & $-285 \pm 220$ & $82 \pm 142$ & $15 \pm 119$ & nnn & 1200 & $\mathrm{R}$ \\
\hline CD-60 1967 & 2002-02-04 & 06:08:09 & $20 \pm 32$ & $49 \pm 59$ & $32 \pm 28$ & nnn & 2700 & $\mathrm{R}$ \\
\hline CD-60 1971 & 2002-02-04 & 06:08:09 & $252 \pm 149$ & $46 \pm 96$ & $101 \pm 80$ & nnn & 2600 & $\mathrm{R}$ \\
\hline V417 Car & 2002-02-04 & $07: 54: 30$ & $-78 \pm 130$ & $-175 \pm 112$ & $-73 \pm 80$ & nnn & 1400 & $\mathrm{R}$ \\
\hline CPD-60 984 & 2002-02-04 & 07:01:42 & $33 \pm 137$ & $170 \pm 103$ & $52 \pm 77$ & nnn & 1500 & $\mathrm{R}$ \\
\hline CPD-60 986 & 2002-02-04 & $07: 01: 42$ & $-62 \pm 160$ & $-47 \pm 153$ & $-38 \pm 109$ & nnn & 1825 & $\mathrm{R}$ \\
\hline V418 Car & 2002-02-05 & 04:19:29 & $-9 \pm 222$ & $98 \pm 172$ & $68 \pm 133$ & $\mathrm{nnn}$ & 1190 & $\mathrm{R}$ \\
\hline \multirow[t]{2}{*}{ CD-60 1974} & 2002-02-05 & $04: 19: 29$ & $-301 \pm 154$ & $-24 \pm 119$ & $-126 \pm 94$ & nnn & 2100 & $\mathrm{R}$ \\
\hline & 2004-03-08 & $05: 25: 21$ & $-168 \pm 128$ & $139 \pm 360$ & $-165 \pm 121$ & dnd & 1350 & \\
\hline CD-60 1975 & 2002-02-04 & 06:08:09 & $0 \pm 122$ & $-116 \pm 100$ & $-48 \pm 76$ & nnn & 2100 & $\mathrm{R}$ \\
\hline CD-60 1976 & 2002-02-04 & 07:01:42 & $-50 \pm 182$ & $-12 \pm 121$ & $-58 \pm 104$ & nnn & 1850 & $\mathrm{R}$ \\
\hline CD-60 1979 & $2002-02-05$ & $07: 45: 43$ & $-210 \pm 269$ & $-109 \pm 276$ & $-162 \pm 191$ & nnn & 1050 & $\mathrm{R}$ \\
\hline CD-60 1978 & 2002-02-04 & $07: 01: 42$ & $145 \pm 216$ & $96 \pm 244$ & $67 \pm 161$ & nnn & 1300 & $\mathrm{R}$ \\
\hline CD-60 1981 & 2002-02-05 & $07: 45: 43$ & $414 \pm 220$ & $-77 \pm 161$ & $167 \pm 141$ & nnn & 1000 & $\mathrm{R}$ \\
\hline V420 Car & 2002-02-05 & 04:19:29 & $-29 \pm 215$ & $-227 \pm 172$ & $-127 \pm 134$ & nnn & 1500 & $\mathrm{R}$ \\
\hline HD 66137 & 2002-02-04 & $07: 01: 42$ & $-124 \pm 160$ & $110 \pm 125$ & $53 \pm 100$ & nnn & 1800 & $\mathrm{R}$ \\
\hline HD 66194 & 2002-02-04 & 06:08:09 & $-183 \pm 97$ & $-237 \pm 353$ & $-197 \pm 93$ & nnn & 600 & $\mathrm{R}$ \\
\hline \multirow{2}{*}{ CD-60 1996} & 2002-02-05 & 05:08:50 & $457 \pm 207$ & $744 \pm 206$ & $631 \pm 159$ & $\mathrm{nDD}$ & 1000 & $\mathrm{R}$ \\
\hline & 2003-02-09 & 06:30:09 & $81 \pm 169$ & $98 \pm 147$ & $108 \pm 120$ & nnn & 1200 & \\
\hline \multirow[t]{2}{*}{ CD-60 1999} & 2003-02-09 & 06:30:09 & $100 \pm 289$ & $-67 \pm 91$ & $-53 \pm 87$ & nnn & 1050 & \\
\hline & 2002-02-05 & 05:08:50 & $80 \pm 233$ & $-289 \pm 205$ & $-83 \pm 149$ & nnn & 1350 & $\mathrm{R}$ \\
\hline [N75] 195 & 2005-01-30 & $07: 12: 58$ & $68 \pm 103$ & $-25 \pm 79$ & $34 \pm 58$ & nnn & 2300 & \\
\hline \multirow[t]{2}{*}{ [N75] 196} & 2004-09-27 & 07:57:06 & $183 \pm 166$ & $444 \pm 386$ & $603 \pm 144$ & nnd & 1400 & \\
\hline & 2005-01-30 & $07: 12: 58$ & $-40 \pm 162$ & $-170 \pm 202$ & $-50 \pm 127$ & nnn & 1450 & \\
\hline \multirow[t]{2}{*}{ NGC 2546272} & 2004-09-27 & $07: 57: 06$ & $26 \pm 96$ & $61 \pm 207$ & $22 \pm 86$ & nnn & 2350 & \\
\hline & 2005-01-30 & $07: 12: 58$ & $188 \pm 126$ & $-21 \pm 125$ & $24 \pm 97$ & nnn & 2000 & \\
\hline CD-37 4353 & 2005-01-29 & $06: 52: 31$ & $-126 \pm 71$ & $-30 \pm 346$ & $-102 \pm 69$ & nnn & 2450 & \\
\hline HD 69003 & 2004-09-27 & 08:53:02 & $-48 \pm 53$ & $-151 \pm 259$ & $-42 \pm 51$ & nnn & 3500 & \\
\hline HD 298537 & 2005-01-30 & $07: 45: 58$ & $-221 \pm 334$ & $-402 \pm 238$ & $-293 \pm 178$ & nnn & 750 & \\
\hline HD 298536 & 2005-01-30 & $07: 45: 58$ & $49 \pm 196$ & $-105 \pm 508$ & $-86 \pm 179$ & nnn & 950 & \\
\hline NGC 3114233 & 2003-02-08 & $07: 15: 26$ & $-123 \pm 96$ & $53 \pm 191$ & $-87 \pm 85$ & nnn & 1850 & \\
\hline CPD-59 1698 & 2003-02-08 & $06: 21: 28$ & $1 \pm 126$ & $-333 \pm 406$ & $-9 \pm 115$ & nnn & 1650 & \\
\hline CPD-59 1700 & 2003-02-08 & $07: 15: 26$ & $287 \pm 161$ & $32 \pm 240$ & $212 \pm 133$ & nnn & 1400 & \\
\hline CPD-59 1703 & $2003-02-08$ & $06: 21: 28$ & $-106 \pm 118$ & $-165 \pm 219$ & $-128 \pm 98$ & nnn & 1550 & \\
\hline HD 298051 & 2002-02-04 & 09:19:41 & $-395 \pm 213$ & $-61 \pm 245$ & $-250 \pm 160$ & nnn & 1150 & $\mathrm{R}$ \\
\hline HD 89901 & 2002-02-05 & $08: 36: 32$ & $-153 \pm 163$ & $250 \pm 156$ & $39 \pm 113$ & nnn & 2050 & $\mathrm{R}$ \\
\hline HD 89900 & 2002-02-05 & 08:36:32 & $-3 \pm 136$ & $-1 \pm 135$ & $0 \pm 96$ & nnn & 2050 & $\mathrm{R}$ \\
\hline HD 89915 & 2002-02-05 & $08: 36: 32$ & $37 \pm 176$ & $-189 \pm 121$ & $-113 \pm 100$ & nnn & 2600 & $\mathrm{R}$ \\
\hline HD 89922 & 2002-02-04 & 09:19:41 & $-47 \pm 130$ & $-23 \pm 112$ & $-46 \pm 84$ & nnn & 1800 & $\mathrm{R}$ \\
\hline HD 298047 & 2002-02-05 & $08: 36: 32$ & $1 \pm 179$ & $154 \pm 233$ & $94 \pm 143$ & nnn & 1550 & $\mathrm{R}$ \\
\hline HD 89938 & 2002-02-05 & $08: 36: 32$ & $-152 \pm 181$ & $-10 \pm 206$ & $-91 \pm 136$ & nnn & 1600 & $\mathrm{R}$ \\
\hline HD 89937 & 2002-02-05 & $08: 36: 32$ & $14 \pm 190$ & $120 \pm 154$ & $71 \pm 120$ & nnn & 1600 & $\mathrm{R}$ \\
\hline HD 89956 & 2002-02-05 & 09:20:10 & $-374 \pm 337$ & $20 \pm 222$ & $-81 \pm 185$ & nnn & 1350 & $\mathrm{R}$ \\
\hline CPD-51 3249 & 2003-02-09 & $08: 13: 16$ & $-54 \pm 200$ & $-16 \pm 173$ & $-51 \pm 138$ & nnn & 1300 & \\
\hline HD 298053 & 2003-02-09 & $08: 13: 16$ & $60 \pm 98$ & $105 \pm 111$ & $116 \pm 84$ & nnn & 1700 & \\
\hline HD 303107 & 2005-01-30 & 09:16:30 & $-253 \pm 143$ & $915 \pm 430$ & $-190 \pm 133$ & nnn & 1350 & \\
\hline HD 305535 & 2004-05-28 & 00:02:29 & $-26 \pm 64$ & $225 \pm 123$ & $30 \pm 55$ & nnn & 3000 & \\
\hline HD 305543 & 2004-05-28 & 00:02:29 & $-4 \pm 224$ & $-149 \pm 121$ & $-105 \pm 90$ & nnn & 2700 & \\
\hline HD 96653 & 2004-07-12 & $23: 52: 56$ & $87 \pm 45$ & $824 \pm 486$ & $96 \pm 44$ & nnn & 1600 & \\
\hline NGC 3532447 & 2004-07-12 & $23: 52: 56$ & $-70 \pm 101$ & $456 \pm 324$ & $-3 \pm 95$ & nnn & 2000 & \\
\hline
\end{tabular}


S. Bagnulo et al.: A survey of magnetic fields in open cluster A- and B-type stars with FORS1. I., Online Material p 13

Table A.4. continued.

\begin{tabular}{|c|c|c|c|c|c|c|c|c|}
\hline ID1 & Date & UT & $\begin{array}{c}\left\langle B_{z}\right\rangle(\mathrm{G}) \\
\text { Balmer lines }\end{array}$ & $\begin{array}{c}\left\langle B_{z}\right\rangle(\mathrm{G}) \\
\text { metal lines }\end{array}$ & $\begin{array}{c}\left\langle B_{z}\right\rangle(\mathrm{G}) \\
\text { full spectrum }\end{array}$ & Flag & SNR & Note \\
\hline HD 96790 & $2004-07-12$ & $23: 52: 56$ & $-168 \pm 174$ & $-118 \pm 126$ & $-159 \pm 98$ & nnn & 2150 & \\
\hline CD-47 8868 & 2004-08-03 & 01:01:32 & $3 \pm 89$ & $-284 \pm 290$ & $-16 \pm 84$ & nnn & 2000 & \\
\hline HD 123201B & 2004-07-13 & $00: 32: 44$ & $-109 \pm 96$ & $353 \pm 281$ & $-77 \pm 94$ & nnn & 1700 & \\
\hline HD 127835 & 2003-02-08 & 09:03:56 & $87 \pm 85$ & $-5 \pm 184$ & $77 \pm 78$ & nnn & 2050 & \\
\hline CPD-56 6334 & 2003-02-08 & $08: 22: 37$ & $80 \pm 140$ & $-191 \pm 158$ & $-33 \pm 92$ & nnn & 1950 & \\
\hline NGC 5662126 & 2003-02-08 & $08: 22: 37$ & $233 \pm 141$ & $104 \pm 172$ & $187 \pm 109$ & nnn & 1450 & \\
\hline HD 127900 & 2003-02-08 & 09:03:56 & $-7 \pm 56$ & $236 \pm 126$ & $30 \pm 52$ & nnn & 2700 & \\
\hline HD 127924 & 2003-02-08 & 09:03:56 & $20 \pm 100$ & $-22 \pm 125$ & $25 \pm 88$ & nnn & 2050 & \\
\hline NGC 6087129 & 2004-07-13 & 02:04:24 & $-5 \pm 69$ & $133 \pm 175$ & $46 \pm 62$ & nnn & 2750 & \\
\hline TYC 8719- 717-1 & 2004-07-13 & 02:04:24 & $0 \pm 84$ & $266 \pm 171$ & $51 \pm 74$ & ndn & 2300 & \\
\hline HD 146484 & 2003-02-09 & 08:54:01 & $-247 \pm 100$ & $-316 \pm 258$ & $-222 \pm 98$ & nnd & 1600 & \\
\hline \multirow[t]{2}{*}{ CD-48 11050} & 2004-07-13 & 03:28:19 & $-148 \pm 114$ & $129 \pm 325$ & $-47 \pm 98$ & nnn & 1550 & \\
\hline & $2004-07-24$ & $02: 51: 35$ & $-55 \pm 74$ & $26 \pm 187$ & $-11 \pm 63$ & nnn & 2200 & \\
\hline \multirow[t]{2}{*}{ CD-48 11060} & 2004-07-13 & $03: 28: 19$ & $-143 \pm 172$ & $59 \pm 310$ & $-102 \pm 136$ & nnn & 1250 & \\
\hline & 2004-07-24 & $02: 51: 35$ & $-99 \pm 112$ & $-228 \pm 161$ & $-95 \pm 87$ & nnn & 1850 & \\
\hline HD 323673 & 2004-08-02 & $05: 36: 38$ & $-43 \pm 91$ & $97 \pm 210$ & $1 \pm 81$ & nnn & 2150 & \\
\hline NGC 638328 & 2004-08-03 & $04: 21: 50$ & $134 \pm 145$ & $-30 \pm 448$ & $17 \pm 143$ & nnn & 1250 & \\
\hline NGC 6383700 & 2004-08-03 & $04: 21: 50$ & $177 \pm 186$ & $62 \pm 187$ & $148 \pm 116$ & nnn & 1250 & \\
\hline NGC 638387 & 2004-08-03 & $04: 21: 50$ & $78 \pm 137$ & $68 \pm 175$ & $35 \pm 106$ & nnn & 1250 & \\
\hline HD 317846 & 2004-07-24 & $03: 52: 22$ & $-20 \pm 74$ & $-77 \pm 193$ & $-23 \pm 68$ & nnn & 2700 & \\
\hline NGC 6383102 & 2004-07-24 & $03: 52: 22$ & $163 \pm 109$ & $-221 \pm 327$ & $147 \pm 101$ & nnn & 1750 & \\
\hline HD 317852 & 2004-08-03 & $04: 21: 50$ & $-61 \pm 131$ & $34 \pm 71$ & $-28 \pm 65$ & nnn & 1700 & \\
\hline HD 318108 & 2004-07-25 & $23: 40: 24$ & $-131 \pm 79$ & $-156 \pm 350$ & $-98 \pm 82$ & nnn & 2150 & \\
\hline HD 318109 & 2004-07-25 & $23: 40: 24$ & $67 \pm 87$ & $62 \pm 442$ & $93 \pm 90$ & nnn & 1950 & \\
\hline CD-32 13089 & 2004-08-17 & $01: 20: 22$ & $-19 \pm 94$ & $76 \pm 126$ & $57 \pm 68$ & nnn & 2000 & \\
\hline CD-32 13093 & 2004-08-17 & $01: 20: 22$ & $-156 \pm 113$ & $-406 \pm 344$ & $-245 \pm 106$ & nnn & 1700 & \\
\hline V976 Sco & 2004-08-17 & $01: 20: 22$ & $-62 \pm 214$ & $-199 \pm 279$ & $-84 \pm 142$ & nnn & 950 & \\
\hline HD 318099 & 2004-08-17 & $01: 20: 22$ & $-40 \pm 84$ & $587 \pm 363$ & $-38 \pm 89$ & nnn & 1850 & \\
\hline HD 320765 & $2004-08-23$ & $01: 44: 37$ & $-18 \pm 71$ & $281 \pm 235$ & $0 \pm 65$ & nnn & 2500 & \\
\hline HD 162678 & 2004-08-02 & $06: 36: 03$ & $-2 \pm 36$ & $-14 \pm 132$ & $3 \pm 34$ & nnn & 4150 & \\
\hline HD 162724 & 2004-08-02 & $06: 36: 03$ & $-33 \pm 54$ & $-329 \pm 171$ & $-53 \pm 51$ & nnn & 5200 & \\
\hline BD-19 $5044 \mathrm{~F}$ & 2004-08-23 & $02: 34: 33$ & $94 \pm 100$ & $-2 \pm 255$ & $63 \pm 96$ & nnn & 2050 & \\
\hline BD-19 $5045 ?$ & 2004-08-23 & $02: 34: 33$ & $-12 \pm 74$ & $191 \pm 120$ & $86 \pm 60$ & nnn & 2650 & \\
\hline BD-19 $5044 \mathrm{M}$ & $2004-08-23$ & $02: 34: 33$ & $-50 \pm 127$ & $244 \pm 492$ & $-32 \pm 120$ & nnn & 1450 & \\
\hline \multirow[t]{2}{*}{ HD 170835} & 2004-08-23 & $03: 26: 34$ & $-242 \pm 239$ & $-500 \pm 310$ & $-417 \pm 183$ & nnn & 1500 & \\
\hline & 2004-08-28 & $04: 37: 57$ & $-15 \pm 200$ & $-635 \pm 235$ & $-435 \pm 142$ & $\mathrm{nnD}$ & 2100 & \\
\hline
\end{tabular}


S. Bagnulo et al.: A survey of magnetic fields in open cluster A- and B-type stars with FORS1. I., Online Material p 14

Table A.5. $\left\langle B_{z}\right\rangle$ measurements for stars not of early spectral type.

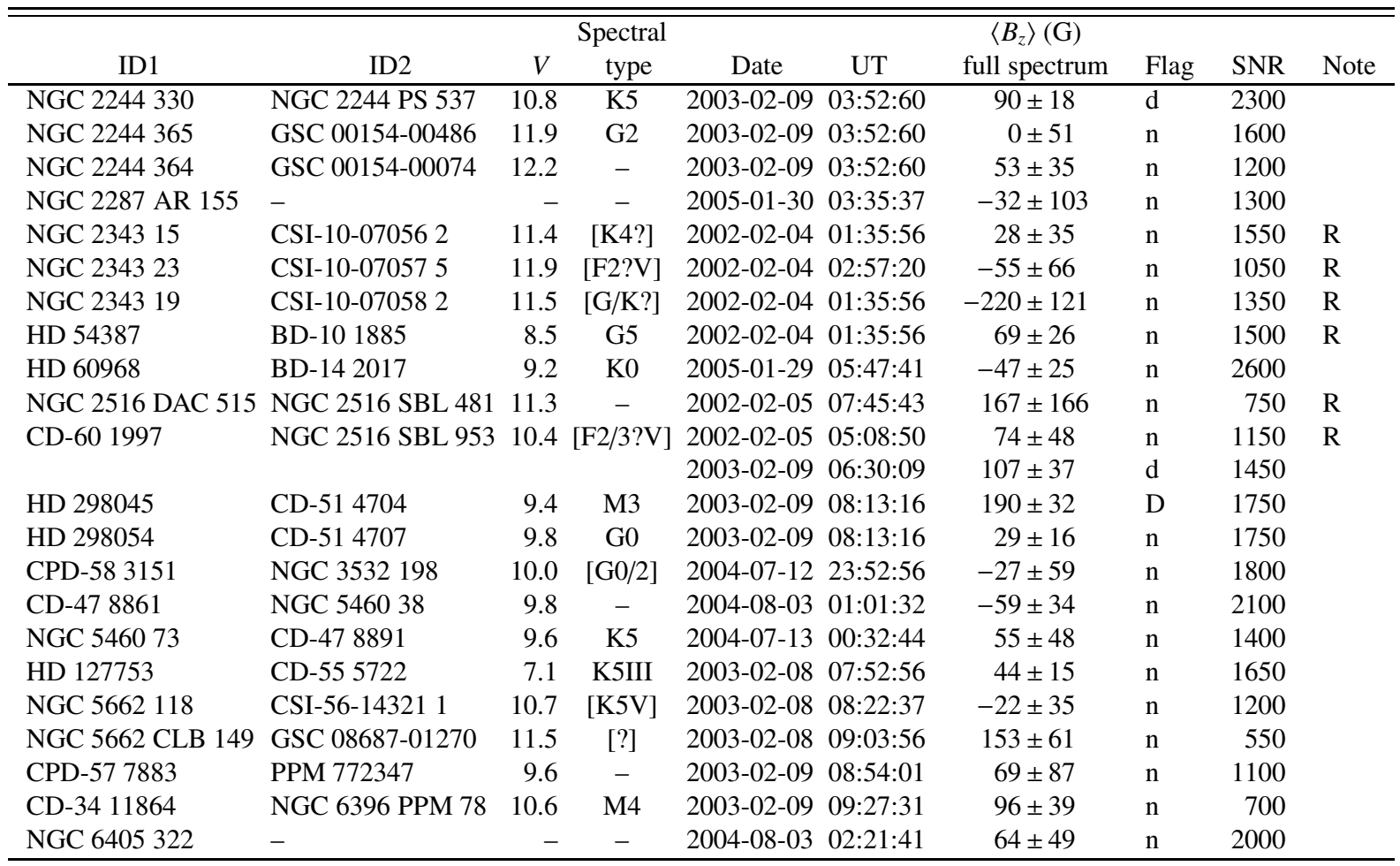

